Supporting Information belonging to the manuscript:

\title{
Factors Controlling the Reactivity and Selectivity of the Diels- Alder Reactions Involving 1,2-Azaborines
}

\author{
Yago García-Rodeja and Israel Fernández*
}

Departamento de Química Orgánica I, Facultad de Ciencias Químicas, Universidad Complutense, 28040 Madrid (Spain).

israel@quim.ucm.es

\section{Contents:}

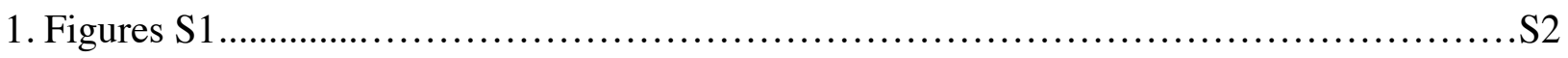

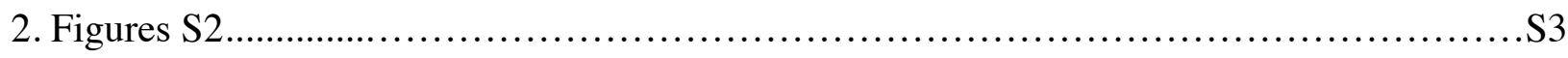

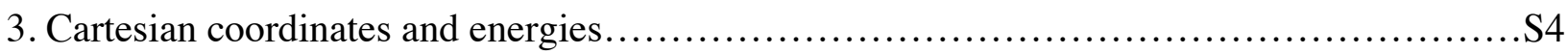




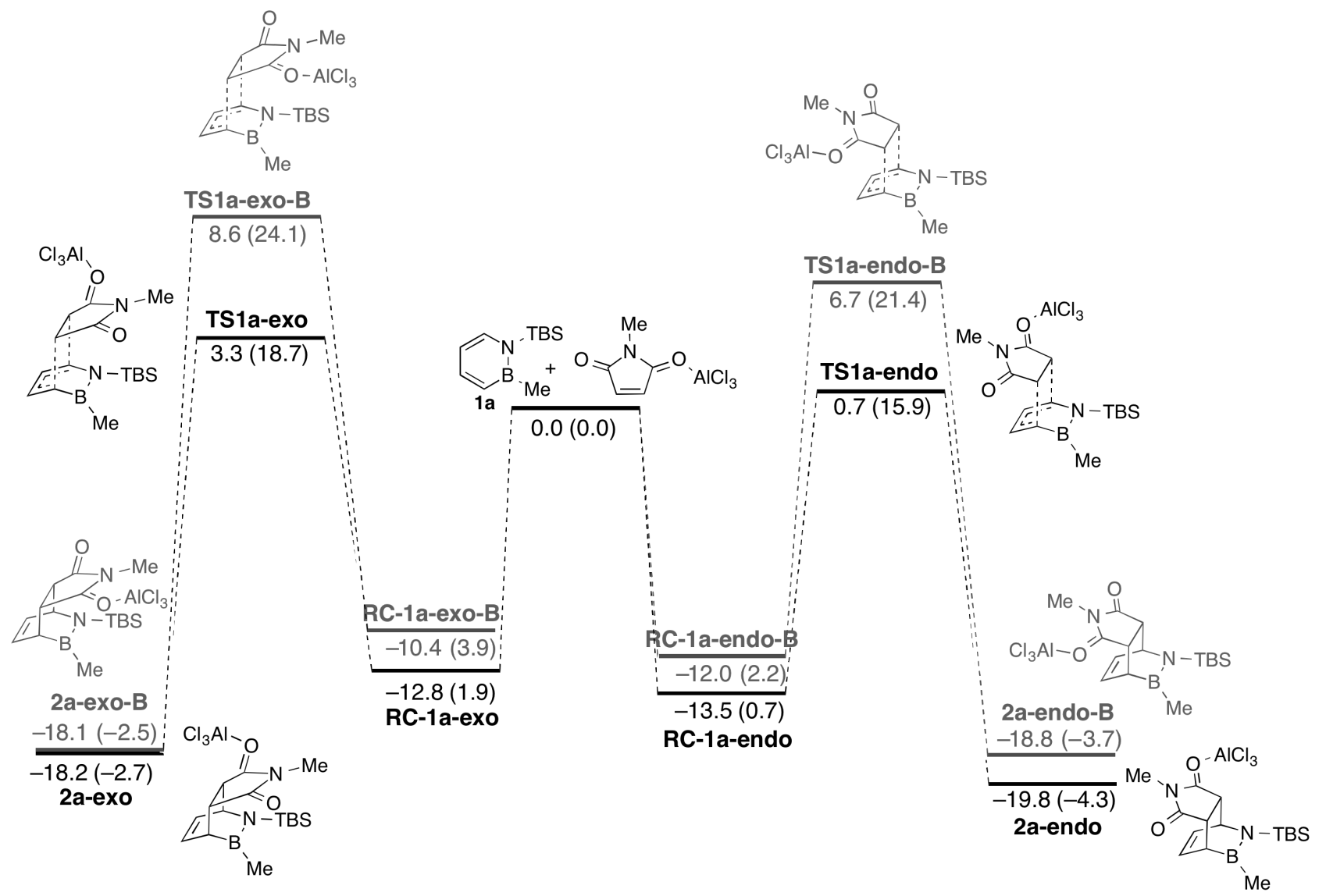

Figure S1. Computed reaction profiles for the uncatalyzed and $\mathrm{AlCl}_{3}$-catalyzed Diels-Alder reactions between $N$-methylmaleimide and 1,2-azaborine 1a. Relative energies (ZPVE included) and free energies (computed at $298 \mathrm{~K}$, within parentheses) are given in $\mathrm{kcal} / \mathrm{mol}$ whereas bond lengths are given in angstroms. All data have been computed at the PCM-(toluene)-B3LYPD3/def2-TZVP//PCM(toluene)B3LYP-D3/def2-SVP level. 


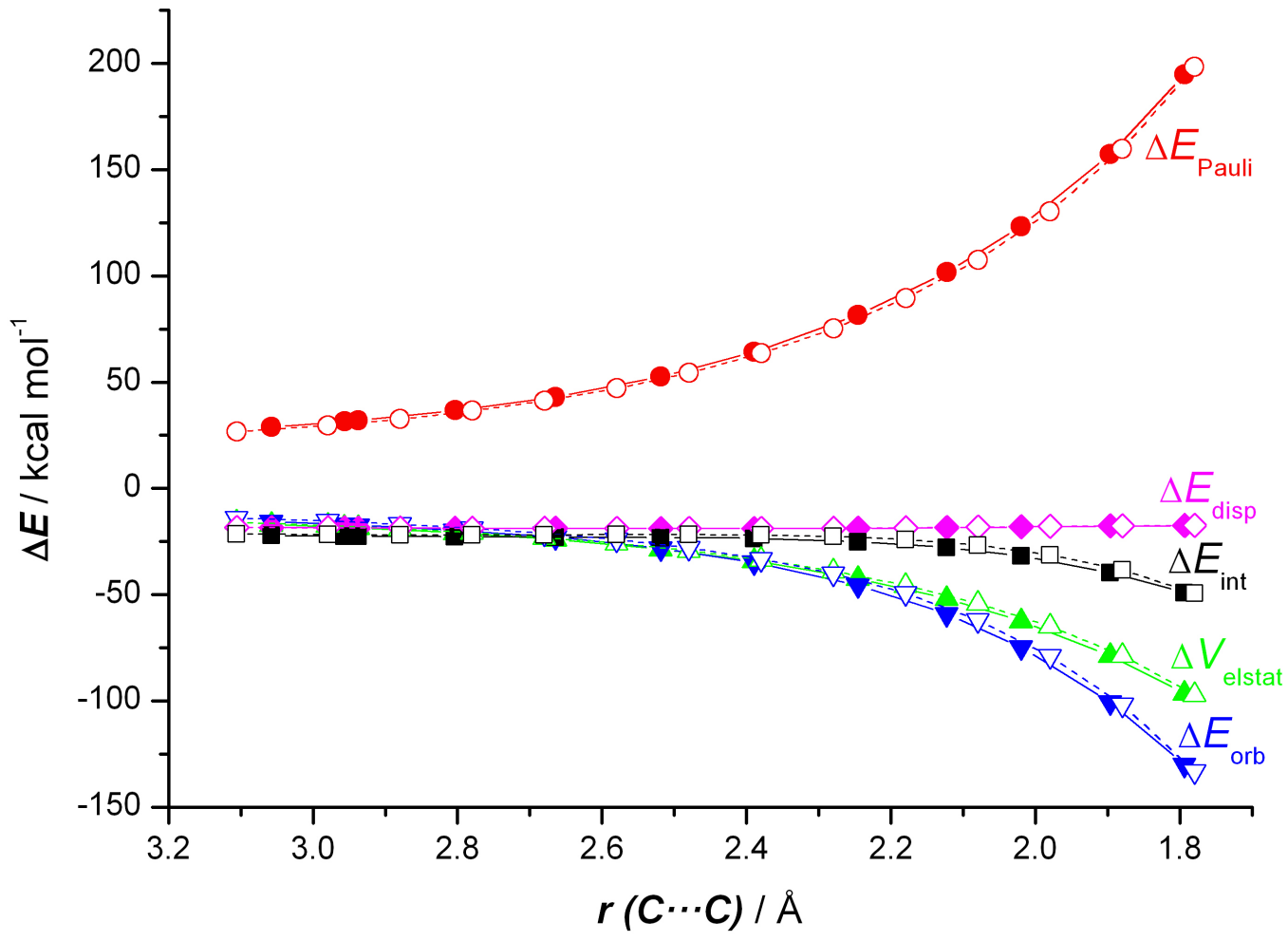

Figure S2. Decomposition of the interaction energy for the $\mathrm{AlCl}_{3}$-catalyzed [4+2]-cycloaddition reactions between $N$-methylmaleimide and 1,2-azaborine 1a (solid lines) and 1c (dotted lines) along the reaction coordinate projected onto the forming $\mathrm{C} \cdots \mathrm{C}$ bond distance. All data have been computed at the BP86-D3/TZ2P//PCM(toluene)-B3LYP-D3/def2-SVP level. Energies are given in $\mathrm{kcal} / \mathrm{mol}$ and bond distances in $\AA$. 
Cartesian coordinates (in $\AA$ ) and total energies (in a.u., noncorrected ZVPE included) of all the stationary points discussed in the text. All calculations have been performed at the PCM(toluene)B3LYP-D3/def2-TZVP//PCM(toluene)-B3LYP-D3/def2-SVP level.

$\begin{array}{lccr}\text { N-Methylmaleimide+AlCl} & : E=-2022.232321 \\ \mathrm{O} & 0.089121000 & -0.431982000 & -0.000205000 \\ \mathrm{O} & 4.626136000 & -0.191329000 & -0.000022000 \\ \mathrm{~N} & 2.349356000 & -0.673304000 & -0.000020000 \\ \mathrm{C} & 1.602499000 & 1.518518000 & -0.000054000 \\ \mathrm{H} & 0.853208000 & 2.307878000 & -0.000060000 \\ \mathrm{C} & 2.940857000 & 1.587334000 & -0.000017000 \\ \mathrm{H} & 3.585379000 & 2.465049000 & 0.000030000 \\ \mathrm{C} & 3.488943000 & 0.188367000 & -0.000019000 \\ \mathrm{C} & 2.401236000 & -2.127667000 & -0.000218000 \\ \mathrm{H} & 3.458461000 & -2.419802000 & -0.000165000 \\ \mathrm{H} & 1.903602000 & -2.524472000 & -0.896314000 \\ \mathrm{H} & 1.903460000 & -2.524710000 & 0.895692000 \\ \mathrm{C} & 1.226686000 & 0.072835000 & -0.000096000 \\ \mathrm{Al} & -1.738957000 & -0.014923000 & 0.000027000 \\ \mathrm{Cl} & -1.827417000 & 2.128566000 & -0.000526000 \\ \mathrm{Cl} & -2.416064000 & -0.912274000 & 1.806453000 \\ \mathrm{Cl} & -2.416904000 & -0.913154000 & -1.805642000\end{array}$

1a: $\mathrm{E}=-801.575109$

\begin{tabular}{|c|c|c|c|}
\hline $\mathrm{Si}$ & -0.849227000 & 0.025404000 & 0.754562000 \\
\hline $\mathrm{N}$ & 0.921511000 & -0.101118000 & 0.332674000 \\
\hline B & 1.783623000 & 1.013596000 & -0.069144000 \\
\hline C & 1.433728000 & -1.382884000 & 0.327552000 \\
\hline $\mathrm{H}$ & 0.760167000 & -2.188046000 & 0.626575000 \\
\hline C & 1.298350000 & 2.530192000 & -0.099879000 \\
\hline $\mathrm{H}$ & 0.339014000 & 2.688981000 & -0.615367000 \\
\hline $\mathrm{H}$ & 2.048866000 & 3.156031000 & -0.609020000 \\
\hline $\mathrm{H}$ & 1.180472000 & 2.940036000 & 0.918307000 \\
\hline $\mathrm{C}$ & 3.214911000 & 0.642845000 & -0.454415000 \\
\hline $\mathrm{H}$ & 3.939148000 & 1.404384000 & -0.766395000 \\
\hline C & 3.624936000 & -0.668691000 & -0.421697000 \\
\hline $\mathrm{H}$ & 4.646953000 & -0.956560000 & -0.697097000 \\
\hline C & 2.720324000 & -1.695404000 & -0.026572000 \\
\hline $\mathrm{H}$ & 3.035156000 & -2.740546000 & 0.002003000 \\
\hline C & -1.256136000 & -1.360685000 & 1.971128000 \\
\hline $\mathrm{H}$ & -1.274551000 & -2.362982000 & 1.517600000 \\
\hline $\mathrm{H}$ & -2.254807000 & -1.173236000 & 2.397248000 \\
\hline $\mathrm{H}$ & -0.533354000 & -1.372704000 & 2.802958000 \\
\hline C & -1.258717000 & 1.640541000 & 1.626120000 \\
\hline $\mathrm{H}$ & -0.521743000 & 1.860144000 & 2.414492000 \\
\hline $\mathrm{H}$ & -2.242091000 & 1.522222000 & 2.110017000 \\
\hline $\mathrm{H}$ & -1.312288000 & 2.507275000 & 0.954706000 \\
\hline $\mathrm{C}$ & -1.864511000 & -0.192988000 & -0.859867000 \\
\hline $\mathrm{C}$ & -1.522965000 & -1.543477000 & -1.520677000 \\
\hline $\mathrm{H}$ & -1.740623000 & -2.399200000 & -0.860604000 \\
\hline $\mathrm{H}$ & -0.461840000 & -1.601755000 & -1.811230000 \\
\hline $\mathrm{H}$ & -2.123914000 & -1.679453000 & -2.437927000 \\
\hline C & -3.364103000 & -0.165677000 & -0.495990000 \\
\hline $\mathrm{H}$ & -3.980191000 & -0.293897000 & -1.404366000 \\
\hline $\mathrm{H}$ & -3.660770000 & 0.790269000 & -0.033222000 \\
\hline $\mathrm{H}$ & -3.636348000 & -0.976354000 & 0.199720000 \\
\hline $\mathrm{C}$ & -1.561620000 & 0.940616000 & -1.858144000 \\
\hline $\mathrm{H}$ & -0.498826000 & 0.959030000 & -2.146979000 \\
\hline
\end{tabular}




$\begin{array}{llll}\mathrm{H} & -1.820437000 & 1.931947000 & -1.452091000 \\ \mathrm{H} & -2.152684000 & 0.802279000 & -2.781554000\end{array}$

RC-1a-endo: $E=-2823.828977$

$\mathrm{Si}$

o

0

$\mathrm{N}$

$\mathrm{N}$

B

C

$\mathrm{H}$

C

$\mathrm{H}$

$\mathrm{H}$

$\mathrm{H}$

C

$\mathrm{H}$

C

$\mathrm{H}$

C

$\mathrm{H}$

C

$\mathrm{H}$

C

$\mathrm{H}$

C

C

$\mathrm{H}$

$\mathrm{H}$

$\mathrm{H}$

C

$\mathrm{H}$

$\mathrm{H}$

$\mathrm{H}$

C

$\mathrm{H}$

$\mathrm{H}$

$\mathrm{H}$

C

C

$\mathrm{H}$

$\mathrm{H}$

$\mathrm{H}$

C

$\mathrm{H}$

$\mathrm{H}$

$\mathrm{H}$

C

$\mathrm{H}$

$\mathrm{H}$

$\mathrm{H}$

Al

$\mathrm{Cl}$

Cl

$\mathrm{Cl}$
$-2.814219000$

2.854304000

1. 461081000

$-1.809428000$

2.383091000

$-2.053845000$

$-0.779187000$

$-0.587532000$

$-3.159841000$

$-4.162030000$

$-3.246124000$

$-2.904392000$

$-1.146283000$

$-1.252336000$

$-0.184999000$

0.453412000

0.021847000

0.805858000

1.007298000

0.648359000

0.567125000

$-0.234666000$

1. 448544000

3.408505000

3.419041000

3. 182976000

4. 389013000

2.146052000

$-1.708629000$

$-1.430442000$

$-2.253866000$

$-0.774935000$

$-3.422763000$

$-2.610115000$

$-3.749145000$

$-4.266879000$

$-4.249780000$

$-3.674575000$

$-3.035368000$

$-3.079688000$

$-4.495292000$

$-5.076047000$

$-5.909573000$

$-5.518269000$

$-4.471928000$

$-5.159817000$

$-4.611834000$

$-5.617504000$

$-5.984663000$

2.932353000

2.008566000

5.013759000

1.806039000
$-0.771473000$

0.199433000

4.486634000

0.526546000

2. 427438000

1. 970773000

0.056784000

$-1.016708000$

2.641925000

2. 202216000

3.716450000

2.558197000

2.831546000

3. 920998000

2.245592000

2.844047000

0.846635000

0.354805000

1. 114429000

0.192649000

2.377912000

2.753066000

3. 288447000

2.868102000

3.964997000

2.514753000

2.477980000

1. 147676000

$-2.267714000$

$-2.803735000$

$-2.977382000$

$-2.002686000$

$-0.189086000$

0.288166000

$-1.076483000$

0.510888000

$-1.223772000$

$-1.723161000$

$-2.612039000$

$-0.946993000$

$-2.006096000$

$-2.349933000$

$-2.646514000$

$-2.033260000$

$-3.252610000$

$-0.010383000$

0.815576000

0.375843000

$-0.295162000$

$-1.649489000$

$-2.175987000$

$-2.073881000$

$-2.310559000$
0.620940000

$-0.060904000$

0.568862000

$-0.203458000$

$-0.004113000$

$-0.151405000$

$-0.974753000$

$-0.968727000$

0.769884000

0.656974000

0.544358000

1.840502000

$-1.039488000$

$-1.074325000$

$-1.835803000$

$-2.495898000$

$-1.776487000$

$-2.353580000$

1.296462000

1.744216000

1.452685000

2.083815000

0.653207000

$-0.932128000$

$-0.916274000$

$-1.949463000$

$-0.626142000$

0.356068000

0.909413000

$-0.009966000$

1.552314000

1. 428864000

2. 301262000

2.871677000

2.867438000

2.251066000

$-0.566947000$

$-1.907402000$

$-1.782650000$

$-2.415455000$

$-2.590616000$

0.089700000

$-0.572106000$

1.048832000

0.276863000

$-0.832082000$

$-1.312780000$

0.093177000

$-1.509570000$

0.026680000

1.895248000

$-0.065789000$

$-1.674032000$

0.516143000

0.089358000

0.245891000

$-0.039421000$

TS-1a-endo: $E=-2823.806354$

$\mathrm{Si}$

O

0

N
$-2.823204000$

2. 994568000

1. 808126000

$-1.756488000$
$-0.877455000$

0.094321000

4. 496763000

0.527980000 


\begin{tabular}{|c|c|}
\hline $\mathrm{N}$ & 2.581781000 \\
\hline B & -1.865452000 \\
\hline C & -0.760242000 \\
\hline $\mathrm{H}$ & -0.545113000 \\
\hline $\mathrm{C}$ & -2.869240000 \\
\hline $\mathrm{H}$ & -3.899400000 \\
\hline $\mathrm{H}$ & -2.888995000 \\
\hline $\mathrm{H}$ & -2.567918000 \\
\hline $\mathrm{C}$ & -0.766585000 \\
\hline $\mathrm{H}$ & -0.903583000 \\
\hline $\mathrm{C}$ & -0.213860000 \\
\hline $\mathrm{H}$ & 0.243676000 \\
\hline $\mathrm{C}$ & -0.112811000 \\
\hline $\mathrm{H}$ & 0.489970000 \\
\hline $\mathrm{C}$ & 0.994560000 \\
\hline $\mathrm{H}$ & 0.573996000 \\
\hline $\mathrm{C}$ & 0.519322000 \\
\hline $\mathrm{H}$ & 0.102931000 \\
\hline $\mathrm{C}$ & 1.687640000 \\
\hline $\mathrm{C}$ & 3.781691000 \\
\hline $\mathrm{H}$ & 3.836594000 \\
\hline $\mathrm{H}$ & 3.743557000 \\
\hline $\mathrm{H}$ & 4.672774000 \\
\hline $\mathrm{C}$ & 2.212644000 \\
\hline $\mathrm{C}$ & -1.808519000 \\
\hline $\mathrm{H}$ & -1.562701000 \\
\hline $\mathrm{H}$ & -2.396507000 \\
\hline $\mathrm{H}$ & -0.864094000 \\
\hline $\mathrm{C}$ & -3.334548000 \\
\hline $\mathrm{H}$ & -2.469163000 \\
\hline $\mathrm{H}$ & -3.719837000 \\
\hline $\mathrm{H}$ & -4.119206000 \\
\hline C & -4.311237000 \\
\hline $\mathrm{C}$ & -3.804311000 \\
\hline $\mathrm{H}$ & -3.241033000 \\
\hline $\mathrm{H}$ & -3.155373000 \\
\hline $\mathrm{H}$ & -4.657851000 \\
\hline $\mathrm{C}$ & -5.213717000 \\
\hline $\mathrm{H}$ & -6.081905000 \\
\hline $\mathrm{H}$ & -5.608338000 \\
\hline $\mathrm{H}$ & -4.682813000 \\
\hline $\mathrm{C}$ & -5.126062000 \\
\hline $\mathrm{H}$ & -4.528563000 \\
\hline $\mathrm{H}$ & -5.526171000 \\
\hline $\mathrm{H}$ & -5.989210000 \\
\hline Al & 2.805952000 \\
\hline $\mathrm{Cl}$ & 1.710707000 \\
\hline $\mathrm{Cl}$ & 4.781238000 \\
\hline $\mathrm{Cl}$ & 1.66523500 \\
\hline
\end{tabular}

2.341396000

1. 926403000

0.214135000

$-0.843317000$

2. 468182000

2. 113559000

3.568197000

2.131646000

2.863743000

3. 941935000

2.476527000

3. 214002000

1.130756000

0.717104000

1.144906000

0.314011000

2. 513156000

2. 971105000

3. 300086000

2. 625491000

3. 712475000

2. 123339000

2. 274058000

1.076702000

$-2.449599000$

$-2.748511000$

$-3.265904000$

$-2.370132000$

$-0.641774000$

$-0.373430000$

$-1.607065000$

0.112363000

$-0.914698000$

$-1.116188000$

$-2.055832000$

$-0.288101000$

$-1.158337000$

$-2.101902000$

$-2.164854000$

$-1.994614000$

$-3.065891000$

0.390104000

1.266315000

0.576786000

0.335421000

$-1.709715000$

$-2.244237000$

$-2.507664000$

$-2.145143000$
$-0.112127000$

0.365004000

$-0.913521000$

$-1.073791000$

1. 449580000

1. 301478000

1. 464531000

2. 457136000

$-0.240390000$

$-0.103388000$

$-1.521669000$

$-2.186443000$

$-1.771429000$

$-2.581808000$

1.020609000

1. 576183000

0.961163000

1. 865201000

0.356676000

$-0.874852000$

$-1.014290000$

$-1.852931000$

$-0.334735000$

0.338930000

0.388920000

$-0.640669000$

0.838954000

0.949482000

2. 306062000

2. 932417000

2. 672938000

2. 451405000

$-0.688965000$

$-2.131221000$

$-2.249030000$

$-2.461072000$

$-2.830817000$

$-0.288822000$

$-0.968844000$

0.735152000

$-0.348282000$

$-0.618880000$

$-0.917803000$

0.390914000

$-1.305802000$

0.076882000

1. 859040000

0.023009000

$-1.709748000$

2a-endo: $E=-2823.839027$

$\begin{array}{lr}\text { Si } & -2.729468000 \\ \mathrm{O} & 2.689621000 \\ \mathrm{O} & 1.729211000 \\ \mathrm{~N} & -1.773842000 \\ \mathrm{~N} & 2.462332000 \\ \mathrm{~B} & -2.147403000 \\ \mathrm{C} & -0.434283000 \\ \mathrm{H} & -0.202706000 \\ \mathrm{C} & -3.491748000 \\ \mathrm{H} & -4.304583000 \\ \mathrm{H} & -3.860404000 \\ \mathrm{H} & -3.287320000\end{array}$

$-0.813997000$

0.122990000

4. 532219000

0.643900000

2.366070000

2. 009251000

0.434683000

$-0.629212000$

2.650243000

1. 955140000

3. 361554000

3.259626000
0.717074000 $-0.247768000$

0.228741000

0.264920000

$-0.113194000$

0.304444000

$-0.350938000$

$-0.440794000$

0.813028000

1.052213000

0.052747000

1.713001000 


$\begin{array}{ll}\mathrm{C} & -0.958152000 \\ \mathrm{H} & -1.169737000 \\ \mathrm{C} & -0.611919000 \\ \mathrm{H} & -0.595982000 \\ \mathrm{C} & -0.332606000 \\ \mathrm{H} & -0.029774000 \\ \mathrm{C} & 0.598119000 \\ \mathrm{H} & 0.605962000 \\ \mathrm{C} & 0.248722000 \\ \mathrm{H} & 0.009153000 \\ \mathrm{C} & 1.507911000 \\ \mathrm{C} & 3.775022000 \\ \mathrm{H} & 3.992024000 \\ \mathrm{H} & 3.770008000 \\ \mathrm{H} & 4.526572000 \\ \mathrm{C} & 1.995669000 \\ \mathrm{C} & -1.537585000 \\ \mathrm{H} & -0.922890000 \\ \mathrm{H} & -2.121604000 \\ \mathrm{H} & -0.847395000 \\ \mathrm{C} & -3.836742000 \\ \mathrm{H} & -3.302645000 \\ \mathrm{H} & -4.116028000 \\ \mathrm{H} & -4.765225000 \\ \mathrm{C} & -3.759981000 \\ \mathrm{C} & -2.818635000 \\ \mathrm{H} & -2.058954000 \\ \mathrm{H} & -2.290949000 \\ \mathrm{H} & -3.395736000 \\ \mathrm{C} & -4.529294000 \\ \mathrm{H} & -5.142086000 \\ \mathrm{H} & -5.213593000 \\ \mathrm{H} & -3.848507000 \\ \mathrm{C} & -4.757891000 \\ \mathrm{H} & -4.245195000 \\ \mathrm{H} & -5.479700000 \\ \mathrm{H} & -5.340510000 \\ \mathrm{Al} & 2.745489000 \\ \mathrm{Cl} & 2.056245000 \\ \mathrm{Cl} & 4.792078000 \\ \mathrm{Cl} & 1.409693000 \\ \mathrm{H} & \\ \mathrm{H} & \\ \mathrm{H} & -100\end{array}$

$\begin{array}{rr}2.958064000 & -0.241609000 \\ 4.033947000 & -0.210695000 \\ 2.452412000 & -1.623433000 \\ 3.119645000 & -2.489074000 \\ 1.141333000 & -1.686823000 \\ 0.600848000 & -2.585397000 \\ 1.128387000 & 0.607109000 \\ 0.597810000 & 1.570868000 \\ 2.627181000 & 0.711394000 \\ 2.940004000 & 1.738022000 \\ 3.354075000 & 0.280296000 \\ 2.686197000 & -0.657381000 \\ 3.733250000 & -0.414916000 \\ 2.552350000 & -1.749163000 \\ 2.018640000 & -0.216559000 \\ 1.120705000 & 0.053852000 \\ -2.179008000 & 1.223557000 \\ -2.570243000 & 0.399575000 \\ -3.020355000 & 1.631106000 \\ -1.839854000 & 2.011410000 \\ -0.442035000 & 2.197009000 \\ 0.166121000 & 2.944357000 \\ -1.394510000 & 2.675852000 \\ 0.081950000 & 1.929863000 \\ -1.346761000 & -0.810137000 \\ -1.602175000 & -2.004240000 \\ -2.370115000 & -1.786054000 \\ -0.683960000 & -2.307493000 \\ -1.951123000 & -2.879809000 \\ -2.639618000 & -0.472350000 \\ -2.958968000 & -1.334908000 \\ -2.503290000 & 0.381932000 \\ -3.471927000 & -0.229758000 \\ -0.235607000 & -1.186797000 \\ 0.710486000 & -1.424976000 \\ -0.032881000 & -0.378828000 \\ -0.528148000 & -2.079266000 \\ -1.707933000 & 0.060459000 \\ -2.2 .56349300000 & 2.086683000 \\ -2.550000 & -1.385887000\end{array}$

RC-1a-exo: $E=-2823.827904$

Si $\quad 2.319198000$

O $\quad-2.245436000$

O 1.104545000

$\mathrm{N} \quad 1.609073000$

N $\quad-0.554726000$

B $\quad 2.247572000$

C $\quad 0.505441000$

$\mathrm{H} \quad 0.035234000$

C $\quad 3.463055000$

$\mathrm{H} \quad 4.293402000$

$\mathrm{H} \quad 3.878955000$

$\mathrm{H} \quad 3.126363000$

C $\quad 1.650470000$

$\mathrm{H} \quad 2.079143000$

C $\quad 0.568403000$

$\mathrm{H} \quad 0.133545000$

C $\quad-0.033656000$

$\mathrm{H} \quad-0.922823000$

C $\quad-1.280235000$

$\mathrm{H} \quad-1.917568000$
$-0.774254000$

0.312465000

3.225096000

0.292351000

1.610800000

1.502395000

$-0.217980000$

$-1.104302000$

2. 237088000

1.579397000

3.010989000

2.749359000

2.040882000

2. 917128000

1. 423442000

1.786996000

0.305220000

$-0.170425000$

2. 168124000

2.100393000
$-0.682491000$

$-0.887437000$

$-1.883640000$

0.630911000

$-1.700939000$

1.168681000

1. 264354000

0.835311000

0.461526000

0.168693000

1. 126024000

$-0.456811000$

2. 473086000

2. 972497000

3. 056610000

3.994497000

2. 423671000

2. 840312000

0.417378000

1.293397000 


$\begin{array}{lr}\mathrm{C} & -0.276565000 \\ \mathrm{H} & 0.111582000 \\ \mathrm{C} & 0.233568000 \\ \mathrm{C} & -0.471206000 \\ \mathrm{H} & 0.478123000 \\ \mathrm{H} & -0.537645000 \\ \mathrm{H} & -1.306095000 \\ \mathrm{C} & -1.437538000 \\ \mathrm{C} & 0.919508000 \\ \mathrm{H} & 0.651280000 \\ \mathrm{H} & 1.249085000 \\ \mathrm{H} & -0.007046000 \\ \mathrm{C} & 3.101297000 \\ \mathrm{H} & 2.548235000 \\ \mathrm{H} & 3.105165000 \\ \mathrm{H} & 4.140598000 \\ \mathrm{C} & 3.583830000 \\ \mathrm{C} & 2.895022000 \\ \mathrm{H} & 2.035424000 \\ \mathrm{H} & 2.537032000 \\ \mathrm{H} & 3.608449000 \\ \mathrm{C} & 4.094471000 \\ \mathrm{H} & 4.825067000 \\ \mathrm{H} & 4.602507000 \\ \mathrm{H} & 3.279915000 \\ \mathrm{C} & 4.777653000 \\ \mathrm{H} & 4.464250000 \\ \mathrm{H} & 5.328821000 \\ \mathrm{H} & 5.493094000 \\ \mathrm{Al} & -3.396938000 \\ \mathrm{Cl} & -2.237874000 \\ \mathrm{Cl} & -5.079675000 \\ \mathrm{Cl} & -3.792742000 \\ & \\ \mathrm{H} & \\ \mathrm{H} & -1000\end{array}$

$\begin{array}{rr}3.023414000 & 0.133657000 \\ 3.845648000 & 0.728300000 \\ 2.709050000 & -1.232023000 \\ 1.069320000 & -3.048091000 \\ 0.539111000 & -3.195701000 \\ 1.891099000 & -3.774420000 \\ 0.373512000 & -3.193381000 \\ 1.258560000 & -0.739133000 \\ -1.805586000 & -1.417475000 \\ -2.669345000 & -0.792445000 \\ -2.191772000 & -2.395097000 \\ -1.237228000 & -1.576285000 \\ 0.251151000 & -2.052722000 \\ 1.179832000 & -2.253990000 \\ -0.352056000 & -2.975221000 \\ 0.529512000 & -1.830741000 \\ -1.940250000 & 0.165113000 \\ -2.716827000 & 1.305328000 \\ -3.308188000 & 0.952137000 \\ -2.046219000 & 2.102891000 \\ -3.423189000 & 1.765885000 \\ -2.934394000 & -0.900788000 \\ -3.630669000 & -0.451570000 \\ -2.422500000 & -1.735322000 \\ -3.544965000 & -1.323037000 \\ -1.160401000 & 0.746350000 \\ -0.445993000 & 1.524039000 \\ -0.601786000 & -0.027076000 \\ -1.859921000 & 1.214715000 \\ -0.775854000 & 0.079721000 \\ -2.555060000 & 0.363036000 \\ -0.984204000 & -1.202406000 \\ 0.247018000 & 1.924974000\end{array}$

TS-1a-exo: $E=-2823.802249$

$\begin{array}{lrrr}\mathrm{Si} & 1.985549000 & -1.112567000 & -0.651742000 \\ \mathrm{O} & -2.019331000 & 0.261756000 & -0.762405000 \\ \mathrm{O} & 0.598838000 & 3.921873000 & -1.443669000 \\ \mathrm{~N} & 1.640025000 & 0.310864000 & 0.476966000 \\ \mathrm{~N} & -0.574791000 & 1.925942000 & -1.384845000 \\ \mathrm{~B} & 2.165787000 & 1.668857000 & 0.364537000 \\ \mathrm{C} & 0.858681000 & 0.030808000 & 1.556639000 \\ \mathrm{H} & 0.341504000 & -0.929106000 & 1.568055000 \\ \mathrm{C} & 3.181425000 & 2.148315000 & -0.739553000 \\ \mathrm{H} & 4.010705000 & 1.440399000 & -0.886223000 \\ \mathrm{H} & 3.610902000 & 3.125841000 & -0.475271000 \\ \mathrm{H} & 2.689843000 & 2.277607000 & -1.717440000 \\ \mathrm{C} & 1.591095000 & 2.656202000 & 1.426022000 \\ \mathrm{H} & 1.978521000 & 3.679581000 & 1.421392000 \\ \mathrm{C} & 1.287417000 & 2.095353000 & 2.714739000 \\ \mathrm{H} & 1.270603000 & 2.715398000 & 3.615967000 \\ \mathrm{C} & 0.834751000 & 0.798383000 & 2.743838000 \\ \mathrm{H} & 0.378549000 & 0.352733000 & 3.629321000 \\ \mathrm{C} & -1.076531000 & 1.835244000 & 0.849879000 \\ \mathrm{H} & -1.623842000 & 1.549023000 & 1.741151000 \\ \mathrm{C} & -0.173023000 & 2.931610000 & 0.682661000 \\ \mathrm{H} & -0.339122000 & 3.878239000 & 1.201056000 \\ \mathrm{C} & 0.031205000 & 3.056863000 & -0.821873000 \\ \mathrm{C} & -0.662814000 & 1.668822000 & -2.809599000 \\ \mathrm{H} & 0.192321000 & 2.151932000 & -3.298649000 \\ \mathrm{H} & -1.594235000 & 2.082512000 & -3.227783000 \\ \mathrm{H} & -0.645918000 & 0.587194000 & -2.992660000 \\ \mathrm{C} & -1.297600000 & 1.250684000 & -0.411666000\end{array}$




$\begin{array}{lr}\mathrm{C} & 0.420354000 \\ \mathrm{H} & 0.006102000 \\ \mathrm{H} & 0.644472000 \\ \mathrm{H} & -0.373526000 \\ \mathrm{C} & 2.435019000 \\ \mathrm{H} & 1.757459000 \\ \mathrm{H} & 2.316389000 \\ \mathrm{H} & 3.467163000 \\ \mathrm{C} & 3.418918000 \\ \mathrm{C} & 2.972472000 \\ \mathrm{H} & 2.096494000 \\ \mathrm{H} & 2.724053000 \\ \mathrm{H} & 3.788410000 \\ \mathrm{C} & 3.804725000 \\ \mathrm{H} & 4.635024000 \\ \mathrm{H} & 4.142391000 \\ \mathrm{H} & 2.967642000 \\ \mathrm{C} & 4.643172000 \\ \mathrm{H} & 4.420050000 \\ \mathrm{H} & 5.020811000 \\ \mathrm{H} & 5.469837000 \\ \mathrm{Al} & -3.190264000 \\ \mathrm{Cl} & -3.283940000 \\ \mathrm{Cl} & -5.015160000 \\ \mathrm{Cl} & -2.301729000 \\ & \end{array}$

$-2.137728000$

$-2.487075000$

$-3.030516000$

$-1.581588000$

$-0.519289000$

0.273402000

$-1.376658000$

$-0.155946000$

$-2.082277000$

$-2.647554000$

$-3.310320000$

$-1.850883000$

$-3.241798000$

$-3.249521000$

$-3.828593000$

$-2.895560000$

$-3.948874000$

$-1.169058000$

$-0.322523000$

$-0.759212000$

$-1.740490000$

$-0.878105000$

$-2.555296000$

0.212302000

$-1.370861000$
-0.780333000
0.175846000
-1.386272000
-1.299986000
-2.373680000
-2.719458000
-3.056188000
-2.462693000
0.166552000
1.529561000
1.440232000
2.249753000
1.978007000
-0.768005000
-0.326419000
-1.755859000
-0.926011000
0.372499000
1.042049000
-0.578297000
0.830841000
0.059435000
-1.259787000
0.275173000
1.968277000

$-0.763933000$

$-0.622756000$

$-1.158216000$

0.180464000

$-1.155873000$

0.115453000

1. 306199000

1.387470000

$-0.884808000$

$-1.565330000$

$-0.304885000$

$-1.488908000$

1. 209941000

1. 183958000

2. 549724000

3. 387990000

2. 607475000

3. 494653000

1. 026775000

1. 829829000

0.883874000

1.530164000

$-0.558112000$

$-2.536314000$

$-3.095915000$

$-2.972158000$

$-2.561339000$

$-0.292077000$

$-0.793659000$

0.193791000

$-1.300219000$

$-1.378485000$

$-2.555466000$

$-2.904011000$

$-3.188553000$

$-2.719580000$ 


$\begin{array}{lr}\mathrm{C} & 3.347986000 \\ \mathrm{C} & 2.932320000 \\ \mathrm{H} & 2.036273000 \\ \mathrm{H} & 2.724389000 \\ \mathrm{H} & 3.744565000 \\ \mathrm{C} & 3.666681000 \\ \mathrm{H} & 4.500477000 \\ \mathrm{H} & 3.970471000 \\ \mathrm{H} & 2.806592000 \\ \mathrm{C} & 4.603842000 \\ \mathrm{H} & 4.427627000 \\ \mathrm{H} & 4.953868000 \\ \mathrm{H} & 5.432767000 \\ \mathrm{Al} & -3.179661000 \\ \mathrm{Cl} & -3.372306000 \\ \mathrm{Cl} & -4.871414000 \\ \mathrm{Cl} & -2.347150000\end{array}$

$-2.090023000$

$-2.485136000$

$-3.127056000$

$-1.599936000$

$-3.048237000$

$-3.360383000$

$-3.915089000$

$-3.123739000$

$-4.047806000$

$-1.200624000$

$-0.284675000$

$-0.898299000$

$-1.747282000$

$-0.927760000$

$-2.493136000$

0.359161000

$-1.530763000$
0.075687000

1.507049000

1.518259000

2.129759000

2.001447000

$-0.738872000$

$-0.271697000$

$-1.772313000$

$-0.788821000$

0.143283000

0.729467000

$-0.857226000$

0.628175000

0.144159000

$-1.281479000$

0.355620000

2. 029955000

TS-1a-stepwise: $E=-2823.788365$

Si $\quad-3.624123000 \quad-0.678870000$

$\mathrm{H} \quad 0.874652000$

$\mathrm{N} \quad-2.554501000$

C 2.559216000

B $\quad-1.229420000$

C $\quad-3.047773000$

$\mathrm{H} \quad-4.027436000$

C $\quad-0.641660000$

$\mathrm{H}-0.182478000$

$\mathrm{H} \quad-1.392659000$

$\mathrm{H} \quad 0.170801000$

C $\quad-0.407652000$

0.449304000

$-1.148604000$

$-0.672617000$

$-2.425032000$

$-2.969764000$

1.525647000

1. 340184000

0.468279000

$-0.339731000$

1. 233178000

3.584615000

2.736466000

$-4.507842000$

$-5.279087000$

$-5.007510000$

$-3.785624000$

$-2.561292000$

$-1.703956000$

$-3.182194000$

$-2.177493000$

$-4.864422000$

$-5.708814000$

$-6.272958000$

$-5.092102000$

$-6.447480000$

$-5.791185000$

$-6.530938000$

$-5.231663000$

$-6.354047000$

$-4.143571000$

$-3.487068000$

$-3.534838000$
$-0.230019000$

0.675397000

0.968993000

0.473996000

1.930737000

2.052846000

$-0.925785000$

$-1.425967000$

$-1.621239000$

$-0.826220000$

1.778392000

3.009926000

3.910805000

3.081698000

4.025632000

2.966301000

4.136869000

1.895895000

2.238996000

0.725145000

0.191837000

2.305178000

0.074058000

0.815692000

$-0.737247000$

0.546047000

$-2.089743000$

$-1.718592000$

$-2.700045000$

$-2.745363000$

$-1.189200000$

0.025038000

0.469210000

0.816542000

$-0.284421000$

$-2.269310000$

$-2.593032000$

$-3.165565000$

$-1.897016000$

$-1.766932000$

$-1.024127000$

$-2.651278000$
1.694941000
$-0.726411000$

$-1.506773000$

$-0.038591000$

$-0.820794000$

0.587245000

$-0.114865000$

$-0.584380000$

0.961525000

0.092297000

1. 362847000

1.695674000

0.824859000

1. 502739000

0.919597000

1. 312989000

0.390246000

0.341283000

$-0.453202000$

$-0.198019000$

$-0.796635000$

$-1.452568000$

$-1.139463000$

$-0.837844000$

$-0.430087000$

$-2.209210000$

$-1.952774000$

$-2.762528000$

$-2.894984000$

$-1.347766000$

$-1.931020000$

$-2.023778000$

$-0.555975000$

0.636625000

1.073497000

0.237356000

1. 530451000

1.833651000

0.034904000

0.788024000

$-0.279402000$

$-0.836619000$

1. 869822000

2. 350016000

1. 622621000 


$\begin{array}{lrrr}\mathrm{H} & -4.885802000 & -2.082988000 & 2.623930000 \\ \mathrm{C} & 3.986161000 & 2.854388000 & 0.058086000 \\ \mathrm{H} & 4.306899000 & 2.319083000 & 0.965003000 \\ \mathrm{H} & 4.772991000 & 2.761175000 & -0.704091000 \\ \mathrm{H} & 3.818261000 & 3.913813000 & 0.289698000 \\ \mathrm{Al} & 3.652997000 & -1.401442000 & 0.036623000 \\ \mathrm{Cl} & 2.215634000 & -2.724266000 & -0.883697000 \\ \mathrm{Cl} & 3.046116000 & -0.849356000 & 2.041235000 \\ \mathrm{Cl} & 5.664160000 & -2.098142000 & -0.102359000\end{array}$

zwitterionic intermediate (stepwise): $E=-2823.788728$

Si

$\mathrm{H}$

$\mathrm{N}$

C

B

C
$-3.670704000$

0.849360000

$-2.565474000$

2.567093000

$-1.215357000$

$-3.035584000$

$-4.034630000$

$-0.678480000$

$-0.190340000$

$-1.453042000$

0.114078000

$-0.329245000$

0.497094000

$-1.064852000$

$-0.546461000$

$-2.367205000$

$-2.907171000$

1.582297000

1. 417811000

0.484719000

$-0.270711000$

1.239270000

3.594626000

2.777792000

$-4.553254000$

$-5.319003000$

$-5.060433000$

$-3.830855000$

$-2.625138000$

$-1.767501000$

$-3.255109000$

$-2.244211000$

$-4.904066000$

$-5.724760000$

$-6.281895000$

$-5.093502000$

$-6.467754000$

$-5.852227000$

$-6.589707000$

$-5.308362000$

$-6.417493000$

$-4.183950000$

$-3.515842000$

$-3.588225000$

$-4.926144000$

4.051787000

4.369675000

4.821624000

3.923608000

3.651368000

2.220577000

3. 016960000
$-0.629281000$

$-0.267755000$

0.683954000

0.913892000

0.438834000

1. 941758000

2. 100857000

$-0.976058000$

$-1.463147000$

$-1.667485000$

$-0.900700000$

1. 721124000

1. 588211000

2.969240000

3. 849535000

3. 070791000

4. 018347000

2.924229000

4.089634000

1. 877807000

2.258174000

0.684886000

0.125138000

2.253533000

0.205772000

0.937264000

$-0.572635000$

0.708249000

$-2.023243000$

$-1.635363000$

$-2.598107000$

$-2.715545000$

$-1.171268000$

0.037336000

0.528583000

0.795788000

$-0.292088000$

$-2.209670000$

$-2.556455000$

$-3.098612000$

$-1.790194000$

$-1.812717000$

$-1.100340000$

$-2.692420000$

$-2.153127000$

2. 771795000

2.245707000

2.633505000

3. 840897000

$-1.386957000$

$-2.786493000$

$-0.704267000$
$-0.736164000$

$-1.492823000$

$-0.011594000$

$-0.901738000$

0.559100000

$-0.012400000$

$-0.428804000$

0.933777000

0.071766000

1.292999000

1.692956000

0.741975000

1. 452994000

0.935843000

1.321798000

0.507418000

0.533606000

$-0.444134000$

$-0.137855000$

$-0.750548000$

$-1.452659000$

$-1.151814000$

$-0.959927000$

$-0.504066000$

$-2.174654000$

$-1.875445000$

$-2.767113000$

$-2.838197000$

$-1.419984000$

$-1.991740000$

$-2.118228000$

$-0.658148000$

0.620237000

1.113002000

0.298923000

1.605084000

1.860298000

$-0.021072000$

0.723844000

$-0.380328000$

$-0.869211000$

1.821502000

2. 330751000

1. 530577000

2.564738000

$-0.047756000$

0.866119000

$-0.819909000$

0.164351000

0.032897000

$-0.785194000$

1. 996131000 
1b: $E=-762.263598$

\begin{tabular}{|c|c|}
\hline $\mathrm{Si}$ & 0.773378000 \\
\hline $\mathrm{N}$ & -0.994112000 \\
\hline B & -1.664260000 \\
\hline $\mathrm{C}$ & -1.678841000 \\
\hline $\mathrm{H}$ & -1.136332000 \\
\hline $\mathrm{H}$ & -1.035680000 \\
\hline $\mathrm{C}$ & -3.115239000 \\
\hline $\mathrm{H}$ & -3.710552000 \\
\hline C & -3.715304000 \\
\hline $\mathrm{H}$ & -4.762849000 \\
\hline $\mathrm{C}$ & -2.993152000 \\
\hline $\mathrm{H}$ & -3.474730000 \\
\hline $\mathrm{C}$ & 1.112147000 \\
\hline $\mathrm{H}$ & 1.031248000 \\
\hline $\mathrm{H}$ & 2.136363000 \\
\hline $\mathrm{H}$ & 0.417972000 \\
\hline $\mathrm{C}$ & 1.179295000 \\
\hline $\mathrm{H}$ & 0.499879000 \\
\hline $\mathrm{H}$ & 2.209707000 \\
\hline $\mathrm{H}$ & 1.098842000 \\
\hline $\mathrm{C}$ & 1.766916000 \\
\hline $\mathrm{C}$ & 1.345733000 \\
\hline $\mathrm{H}$ & 1.529967000 \\
\hline $\mathrm{H}$ & 0.278091000 \\
\hline $\mathrm{H}$ & 1.923366000 \\
\hline $\mathrm{C}$ & 3.269680000 \\
\hline $\mathrm{H}$ & 3.868539000 \\
\hline $\mathrm{H}$ & 3.619405000 \\
\hline $\mathrm{H}$ & 3.508680000 \\
\hline C & 1.511451000 \\
\hline $\mathrm{H}$ & 0.447937000 \\
\hline $\mathrm{H}$ & 1.821975000 \\
\hline $\mathrm{H}$ & 2.084835000 \\
\hline
\end{tabular}

0.715434000

0.310679000

$-0.409121000$

0.611301000

1. 152055000

$-0.668518000$

$-0.791051000$

$-1.333050000$

$-0.439171000$

$-0.692194000$

0.265425000

0.541815000

2. 213980000

1.996477000

2. 573960000

3.036184000

1. 155103000

1. 941408000

1. 542807000

0.293234000

$-0.806239000$

$-1.164906000$

$-0.338666000$

$-1.431220000$

$-2.035325000$

$-0.455546000$

$-1.318522000$

$-0.199181000$

0.391415000

$-2.018120000$

$-2.304867000$

$-1.825216000$

$-2.892483000$
0.297143000

0.167539000

1.233720000

$-0.984842000$

$-1.764166000$

2. 230860000

1.012416000

1. 754764000

$-0.178459000$

$-0.382920000$

$-1.183646000$

$-2.123875000$

$-0.794960000$

$-1.871144000$

$-0.606344000$

$-0.556785000$

2. 076629000

2.442851000

2.131084000

2.753697000

$-0.311305000$

$-1.750577000$

$-2.457094000$

$-1.812718000$

$-2.110683000$

$-0.288425000$

$-0.631623000$

0.725540000

$-0.952644000$

0.605580000

0.622191000

1.645300000

0.247863000

\section{RC-1b-endo: $E=-2784.516363$}

\section{$\mathrm{Si}$}

o

O

$\mathrm{N}$

N

B

C

$\mathrm{H}$

$\mathrm{H}$

C

$\mathrm{H}$

C

$\mathrm{H}$

C

$\mathrm{H}$

C

$\mathrm{H}$

C

$\mathrm{H}$

C

C

$\mathrm{H}$

$\mathrm{H}$

$\mathrm{H}$
2.931435000

$-2.775653000$

$-1.388102000$

1. 853116000

$-2.318469000$

2. 090276000

0.820909000

0.642871000

2. 982712000

1.182929000

1. 277781000

0.200165000

$-0.463978000$

0.002832000

$-0.793819000$

$-0.914361000$

$-0.546241000$

$-0.478146000$

0.335220000

$-1.374454000$

$-3.355950000$

$-3.362198000$

$-3.145753000$

$-4.333422000$
$-0.603826000$

0.194477000

4.451190000

0.575416000

2. 420020000

2.003436000

0.110282000

$-0.966573000$

2.398751000

2. 911956000

4.002046000

2.337697000

2. 953599000

0.934755000

0.469738000

1.050916000

0.107951000

2.305923000

2. 651753000

3. 251106000

2. 902148000

3. 997433000

2. 590802000

2. 502955000
$-0.621088000$

0.011758000

$-0.804894000$

0.267761000

$-0.151795000$

0.183768000

1.031360000

1. 056936000

$-0.521880000$

0.999972000

1. 000731000

1. 784959000

2.402602000

1. 782076000

2. 364116000

$-1.363281000$

$-1.757159000$

$-1.577370000$

$-2.211259000$

$-0.834713000$

0.741879000

0.680158000

1. 775996000

0.437998000 
$-2.069551000$

2.023450000

1. 935926000

2.587285000

1. 008836000

3.239466000

2. 283109000

3.812598000

3. 801473000

4.540340000

4.181333000

3. 616371000

3. 580096000

5.100382000

5.391582000

6.335118000

5.661807000

4.873651000

5.350267000

4.792542000

5.638434000

6.281124000

$-2.831054000$

$-1.773485000$

$-4.909869000$

$-1.814708000$
1.125783000

$-2.244892000$

$-2.754697000$

$-2.911725000$

$-2.135276000$

0.087611000

0.267477000

$-0.640873000$

1. 032117000

$-0.796923000$

$-1.199157000$

$-2.144901000$

$-0.425083000$

$-1.343057000$

$-1.909227000$

$-2.041338000$

$-1.668352000$

$-2.882021000$

0.513069000

1. 337499000

0.838467000

0.372147000

$-1.659167000$

$-2.300736000$

$-2.101627000$

$-2.187222000$
-0.442417000
-0.741366000
0.230714000
-1.413375000
-1.155622000
-2.340241000
-2.857779000
-2.936575000
-2.318160000
0.399244000
1.843591000
1.884812000
2.347577000
2.439431000
-0.250328000
0.308968000
-1.292205000
-0.251393000
0.421161000
0.892222000
-0.591678000
0.999371000
0.041158000
-1.718526000
0.015785000
1.851740000

TS-1b-endo: $E=-2784.488462$

\begin{tabular}{|c|c|}
\hline $\mathrm{C}$ & -2.123996000 \\
\hline $\mathrm{N}$ & -2.536306000 \\
\hline C & -1.630103000 \\
\hline C & -0.415307000 \\
\hline C & -0.854838000 \\
\hline $\mathrm{C}$ & -3.777197000 \\
\hline C & 0.819767000 \\
\hline C & 0.252049000 \\
\hline $\mathrm{C}$ & 0.120693000 \\
\hline C & 0.727991000 \\
\hline $\mathrm{N}$ & 1.790194000 \\
\hline B & 1.930644000 \\
\hline O & -1.780070000 \\
\hline 0 & -2.901649000 \\
\hline Al & -2.675278000 \\
\hline $\mathrm{Cl}$ & -1.685789000 \\
\hline $\mathrm{Si}$ & 2.874784000 \\
\hline $\mathrm{C}$ & 4.563570000 \\
\hline C & 5.249806000 \\
\hline $\mathrm{C}$ & 2.065842000 \\
\hline $\mathrm{C}$ & 2.981168000 \\
\hline $\mathrm{C}$ & 4.356246000 \\
\hline $\mathrm{C}$ & 5.462442000 \\
\hline $\mathrm{Cl}$ & -1.400626000 \\
\hline $\mathrm{Cl}$ & -4.626965000 \\
\hline $\mathrm{H}$ & 0.471022000 \\
\hline $\mathrm{H}$ & 2.766323000 \\
\hline $\mathrm{H}$ & 0.956207000 \\
\hline $\mathrm{H}$ & -0.196217000 \\
\hline $\mathrm{H}$ & -0.495673000 \\
\hline $\mathrm{H}$ & -0.429363000 \\
\hline $\mathrm{H}$ & -0.012699000 \\
\hline $\mathrm{H}$ & -3.856625000 \\
\hline $\mathrm{H}$ & -3.778476000 \\
\hline $\mathrm{H}$ & -4.633366000 \\
\hline
\end{tabular}

1.042303000

2.328048000

3.266097000

2.461897000

1.073984000

2.651084000

2.949751000

2.668331000

1. 350653000

0.358251000

0.608084000

1. 956932000

4.463098000

0.066383000

$-1.730900000$

$-1.974687000$

$-0.760067000$

$-0.526421000$

0.784140000

$-2.375569000$

$-0.621182000$

$-0.516803000$

$-1.719798000$

$-2.362068000$

$-2.578777000$

$-0.683866000$

2. 232751000

4. 012368000

3.463439000

1. 010378000

0.220915000

2. 829157000

3. 744463000

2. 233122000

2. 236791000
$-0.424942000$

$-0.106992000$

$-0.604000000$

$-1.084318000$

$-1.021889000$

0.571529000

0.073624000

1. 387898000

1. 735660000

0.922342000

0.098984000

$-0.379682000$

$-0.595253000$

$-0.152408000$

0.016115000

1. 921392000

$-0.484909000$

0.386427000

$-0.043361000$

0.012116000

$-2.351986000$

1. 914096000

$-0.004814000$

$-1.610116000$

$-0.049566000$

1.119919000

$-1.195485000$

$-0.153252000$

1. 989100000

2. 570068000

$-1.538492000$

$-2.035350000$

0.615445000

1. 589050000

0.019941000 


$\begin{array}{ll}\mathrm{H} & 2.004046000 \\ \mathrm{H} & 2.665771000 \\ \mathrm{H} & 1.051408000 \\ \mathrm{H} & 1.979899000 \\ \mathrm{H} & 3.623895000 \\ \mathrm{H} & 3.393550000 \\ \mathrm{H} & 3.895759000 \\ \mathrm{H} & 3.718331000 \\ \mathrm{H} & 5.327073000 \\ \mathrm{H} & 6.449154000 \\ \mathrm{H} & 5.639145000 \\ \mathrm{H} & 5.033708000 \\ \mathrm{H} & 4.675566000 \\ \mathrm{H} & 5.400846000 \\ \mathrm{H} & 6.244329000\end{array}$

-2.514973000
-3.204677000
-2.464833000
-0.743187000
-1.422477000
0.343929000
-1.449870000
0.322328000
-0.410056000
-1.625840000
-1.766171000
-2.684683000
1.673199000
0.838418000
0.862315000
1.102255000
-0.396344000
-0.406666000
-2.796135000
-2.751010000
-2.680590000
2.276993000
2.236758000
2.429866000
0.482589000
-1.092252000
0.310560000
0.261188000
-1.133680000
0.431166000

0.792593000

$-0.292394000$

0.296608000

0.350722000

$-0.108473000$

0.520955000

$-0.335504000$

$-0.502072000$

1. 057291000

$-0.032126000$

0.076908000

$-1.460598000$

$-2.280663000$

$-1.622480000$

$-2.569700000$

0.629251000

1. 564047000

0.818486000

1.873509000

0.337969000

$-0.681495000$

$-0.407314000$

$-1.776839000$

$-0.289106000$

0.044118000

1. 449639000

0.683055000

1. 834649000

2.276382000

2. 125885000

3. 003257000

2.453699000

1. 772252000

$-0.776800000$

$-1.913001000$

$-1.622501000$

$-2.251429000$

$-2.788841000$

$-0.435680000$

$-1.318154000$

0.376477000

$-0.131681000$

$-1.241826000$

$-1.461949000$

$-0.486619000$

$-2.165924000$ 


$\begin{array}{lllr}\mathrm{Al} & 2.384586000 & -1.901128000 & 0.017727000 \\ \mathrm{Cl} & 1.730610000 & -1.973542000 & 2.059403000 \\ \mathrm{Cl} & 4.342046000 & -2.651548000 & -0.327522000 \\ \mathrm{Cl} & 0.907489000 & -2.583255000 & -1.379808000\end{array}$

RC-1b-exo: $E=-2784.514199$

\begin{tabular}{|c|c|c|c|}
\hline Si & 2.438328000 & -0.522121000 & -0.683456000 \\
\hline O & -2.085549000 & 0.290169000 & -0.846893000 \\
\hline O & 0.941247000 & 3.579901000 & -1.679861000 \\
\hline $\mathrm{N}$ & 1.667114000 & 0.373379000 & 0.708912000 \\
\hline $\mathrm{N}$ & -0.540679000 & 1.794458000 & -1.588378000 \\
\hline B & 2.279436000 & 1.576686000 & 1.242464000 \\
\hline C & 0.610619000 & -0.197153000 & 1.364590000 \\
\hline $\mathrm{H}$ & 0.164094000 & -1.094520000 & 0.929767000 \\
\hline $\mathrm{H}$ & 3.193524000 & 2.070926000 & 0.634226000 \\
\hline $\mathrm{C}$ & 1.732964000 & 2.109994000 & 2.557160000 \\
\hline $\mathrm{H}$ & 2.142374000 & 2.996161000 & 3.052997000 \\
\hline $\mathrm{C}$ & 0.688402000 & 1.441898000 & 3.163374000 \\
\hline $\mathrm{H}$ & 0.268953000 & 1.783790000 & 4.116526000 \\
\hline C & 0.105477000 & 0.301599000 & 2.550317000 \\
\hline $\mathrm{H}$ & -0.750325000 & -0.203015000 & 3.001420000 \\
\hline $\mathrm{C}$ & -1.294497000 & 2.148512000 & 0.565548000 \\
\hline $\mathrm{H}$ & -1.913328000 & 1.962327000 & 1.437823000 \\
\hline C & -0.377908000 & 3.107927000 & 0.334807000 \\
\hline $\mathrm{H}$ & -0.055478000 & 3.919169000 & 0.981936000 \\
\hline C & 0.142459000 & 2.935225000 & -1.055464000 \\
\hline $\mathrm{C}$ & -0.415421000 & 1.344758000 & -2.966002000 \\
\hline $\mathrm{H}$ & 0.559650000 & 0.869630000 & -3.133974000 \\
\hline $\mathrm{H}$ & -0.511604000 & 2.209250000 & -3.636910000 \\
\hline $\mathrm{H}$ & -1.214340000 & 0.621587000 & -3.168714000 \\
\hline $\mathrm{C}$ & -1.375063000 & 1.301009000 & -0.647795000 \\
\hline C & 1.105595000 & -1.559774000 & -1.519044000 \\
\hline $\mathrm{H}$ & 0.895329000 & -2.489066000 & -0.969998000 \\
\hline $\mathrm{H}$ & 1.445191000 & -1.838618000 & -2.529449000 \\
\hline $\mathrm{H}$ & 0.144725000 & -1.036302000 & -1.617413000 \\
\hline $\mathrm{C}$ & 3.175142000 & 0.731262000 & -1.873723000 \\
\hline $\mathrm{H}$ & 2.503078000 & 1.581740000 & -2.061057000 \\
\hline $\mathrm{H}$ & 3.385305000 & 0.238125000 & -2.836769000 \\
\hline $\mathrm{H}$ & 4.119866000 & 1.143232000 & -1.490779000 \\
\hline C & 3.787205000 & -1.660339000 & 0.064306000 \\
\hline C & 3.161775000 & -2.594262000 & 1.118775000 \\
\hline $\mathrm{H}$ & 2.364318000 & -3.227608000 & 0.698377000 \\
\hline $\mathrm{H}$ & 2.735146000 & -2.030482000 & 1.963860000 \\
\hline $\mathrm{H}$ & 3.931610000 & -3.270781000 & 1.530907000 \\
\hline $\mathrm{C}$ & 4.386891000 & -2.500684000 & -1.082842000 \\
\hline $\mathrm{H}$ & 5.177863000 & -3.167618000 & -0.695484000 \\
\hline $\mathrm{H}$ & 4.846128000 & -1.868584000 & -1.861207000 \\
\hline $\mathrm{H}$ & 3.630165000 & -3.139310000 & -1.567559000 \\
\hline C & 4.900585000 & -0.827852000 & 0.727966000 \\
\hline $\mathrm{H}$ & 4.516378000 & -0.209820000 & 1.555498000 \\
\hline $\mathrm{H}$ & 5.404223000 & -0.157461000 & 0.013325000 \\
\hline $\mathrm{H}$ & 5.672576000 & -1.496422000 & 1.149512000 \\
\hline Al & -3.186659000 & -0.926755000 & 0.024877000 \\
\hline $\mathrm{Cl}$ & -1.954067000 & -2.671315000 & 0.169395000 \\
\hline $\mathrm{Cl}$ & -4.854176000 & -1.099032000 & -1.282489000 \\
\hline $\mathrm{Cl}$ & -3.632851000 & -0.072030000 & 1.942167000 \\
\hline
\end{tabular}

TS-1b-exo: $E=-2784.485310$

$\begin{array}{lrrr}\mathrm{Si} & 2.147955000 & -0.887833000 & -0.716362000 \\ \mathrm{O} & -1.938130000 & 0.172848000 & -0.738886000 \\ \mathrm{O} & 0.333579000 & 4.014182000 & -1.614513000 \\ \mathrm{~N} & 1.703524000 & 0.505883000 & 0.400917000\end{array}$




\begin{tabular}{|c|c|}
\hline $\mathrm{N}$ & -0.646910000 \\
\hline B & 2.085250000 \\
\hline $\mathrm{C}$ & 0.997791000 \\
\hline $\mathrm{H}$ & 0.541386000 \\
\hline $\mathrm{H}$ & 2.682107000 \\
\hline $\mathrm{C}$ & 1.549334000 \\
\hline $\mathrm{H}$ & 1.872081000 \\
\hline $\mathrm{C}$ & 1.391726000 \\
\hline $\mathrm{H}$ & 1.415022000 \\
\hline $\mathrm{C}$ & 1.023120000 \\
\hline $\mathrm{H}$ & 0.670273000 \\
\hline $\mathrm{C}$ & -1.036144000 \\
\hline $\mathrm{H}$ & -1.540096000 \\
\hline $\mathrm{C}$ & -0.228670000 \\
\hline $\mathrm{H}$ & -0.473329000 \\
\hline $\mathrm{C}$ & -0.116051000 \\
\hline $\mathrm{C}$ & -0.755278000 \\
\hline $\mathrm{H}$ & -0.021136000 \\
\hline $\mathrm{H}$ & -1.764448000 \\
\hline $\mathrm{H}$ & -0.552922000 \\
\hline $\mathrm{C}$ & -1.274781000 \\
\hline $\mathrm{C}$ & 0.651263000 \\
\hline $\mathrm{H}$ & 0.237361000 \\
\hline $\mathrm{H}$ & 0.937777000 \\
\hline $\mathrm{H}$ & -0.162176000 \\
\hline $\mathrm{C}$ & 2.634349000 \\
\hline $\mathrm{H}$ & 1.863295000 \\
\hline $\mathrm{H}$ & 2.743902000 \\
\hline $\mathrm{H}$ & 3.586500000 \\
\hline $\mathrm{C}$ & 3.620660000 \\
\hline $\mathrm{C}$ & 3.184624000 \\
\hline $\mathrm{H}$ & 2.353296000 \\
\hline $\mathrm{H}$ & 2.874293000 \\
\hline $\mathrm{H}$ & 4.027639000 \\
\hline $\mathrm{C}$ & 4.113113000 \\
\hline $\mathrm{H}$ & 4.968643000 \\
\hline $\mathrm{H}$ & 4.453514000 \\
\hline $\mathrm{H}$ & 3.331720000 \\
\hline $\mathrm{C}$ & 4.764748000 \\
\hline $\mathrm{H}$ & 4.457926000 \\
\hline $\mathrm{H}$ & 5.128783000 \\
\hline $\mathrm{H}$ & 5.623731000 \\
\hline Al & -2.996543000 \\
\hline $\mathrm{Cl}$ & -3.011720000 \\
\hline $\mathrm{Cl}$ & -4.885136000 \\
\hline $\mathrm{Cl}$ & -2.013521000 \\
\hline
\end{tabular}

\begin{tabular}{|c|c|}
\hline 1.918614000 & -1.456444000 \\
\hline 1.872464000 & 0.160038000 \\
\hline 0.245686000 & 1.538684000 \\
\hline-0.740728000 & 1.636995000 \\
\hline 2.173423000 & -0.833371000 \\
\hline 2.904699000 & 1.186470000 \\
\hline 3.945317000 & 1.088749000 \\
\hline 2.403318000 & 2.532348000 \\
\hline 3.076585000 & 3.394159000 \\
\hline 1.088367000 & 2.677071000 \\
\hline 0.678365000 & 3.625116000 \\
\hline 1.840120000 & 0.802248000 \\
\hline 1.551189000 & 1.718037000 \\
\hline 3.005009000 & 0.567783000 \\
\hline 3.946559000 & 1.065508000 \\
\hline 3.114308000 & -0.950764000 \\
\hline 1.605294000 & -2.868932000 \\
\hline 2.218593000 & -3.406095000 \\
\hline 1.834186000 & -3.245387000 \\
\hline 0.539376000 & -3.033981000 \\
\hline 1.216514000 & -0.439242000 \\
\hline-2.001704000 & -0.881569000 \\
\hline-2.337100000 & 0.080570000 \\
\hline-2.902615000 & -1.447711000 \\
\hline-1.508357000 & -1.432770000 \\
\hline-0.165360000 & -2.375472000 \\
\hline 0.520607000 & -2.754669000 \\
\hline-0.986840000 & -3.101791000 \\
\hline 0.383011000 & -2.333220000 \\
\hline-1.772964000 & 0.126680000 \\
\hline-2.395298000 & 1.467951000 \\
\hline-3.108895000 & 1.348614000 \\
\hline-1.631370000 & 2.199582000 \\
\hline-2.946763000 & 1.921131000 \\
\hline-2.891144000 & -0.817889000 \\
\hline-3.421314000 & -0.363037000 \\
\hline-2.494618000 & -1.788482000 \\
\hline-3.643541000 & -1.014797000 \\
\hline-0.771195000 & 0.377369000 \\
\hline 0.041793000 & 1.055556000 \\
\hline-0.312304000 & -0.556214000 \\
\hline-1.283374000 & 0.846139000 \\
\hline-1.035461000 & 0.134474000 \\
\hline-2.734894000 & -1.158151000 \\
\hline-0.071314000 & 0.396342000 \\
\hline-1.434056000 & 2.01896000 \\
\hline
\end{tabular}

\section{2b-exo: $E=-2784.518363$}

$\begin{array}{lr}\mathrm{Si} & 2.114935000 \\ \mathrm{O} & -1.817133000 \\ \mathrm{O} & -0.199047000 \\ \mathrm{~N} & 1.620122000 \\ \mathrm{~N} & -1.108213000 \\ \mathrm{~B} & 2.165546000 \\ \mathrm{C} & 0.749957000 \\ \mathrm{H} & 0.383288000 \\ \mathrm{H} & 2.908858000 \\ \mathrm{C} & 1.630138000 \\ \mathrm{H} & 1.997319000 \\ \mathrm{C} & 1.925777000 \\ \mathrm{H} & 2.475786000 \\ \mathrm{C} & 1.463611000 \\ \mathrm{H} & 1.547061000\end{array}$

$\begin{array}{rr}-0.891074000 & -0.881682000 \\ 0.092718000 & -0.570412000 \\ 4.277983000 & -1.250838000 \\ 0.583301000 & 0.026587000 \\ 2.151381000 & -1.167222000 \\ 1.865679000 & -0.163865000 \\ 0.499395000 & 1.230243000 \\ -0.517960000 & 1.391913000 \\ 2.130800000 & -1.070990000 \\ 2.918935000 & 0.921079000 \\ 3.945877000 & 0.812075000 \\ 2.306603000 & 2.275601000 \\ 2.861159000 & 3.039901000 \\ 1.059777000 & 2.445579000 \\ 0.478826000 & 3.365487000\end{array}$




$\begin{array}{lr}\mathrm{C} & -0.470177000 \\ \mathrm{H} & -1.168741000 \\ \mathrm{C} & 0.062200000 \\ \mathrm{H} & -0.389582000 \\ \mathrm{C} & -0.384820000 \\ \mathrm{C} & -1.692556000 \\ \mathrm{H} & -1.170115000 \\ \mathrm{H} & -1.578026000 \\ \mathrm{H} & -2.754372000 \\ \mathrm{C} & -1.201419000 \\ \mathrm{C} & 0.658653000 \\ \mathrm{H} & 0.250396000 \\ \mathrm{H} & 0.978600000 \\ \mathrm{H} & -0.163823000 \\ \mathrm{C} & 2.607581000 \\ \mathrm{H} & 1.800327000 \\ \mathrm{H} & 2.784489000 \\ \mathrm{H} & 3.521818000 \\ \mathrm{C} & 3.588654000 \\ \mathrm{C} & 3.123914000 \\ \mathrm{H} & 2.320032000 \\ \mathrm{H} & 2.758949000 \\ \mathrm{H} & 3.964473000 \\ \mathrm{C} & 4.121105000 \\ \mathrm{H} & 4.964119000 \\ \mathrm{H} & 4.491689000 \\ \mathrm{H} & 3.351772000 \\ \mathrm{C} & 4.712110000 \\ \mathrm{H} & 4.379484000 \\ \mathrm{H} & 5.080237000 \\ \mathrm{H} & 5.573916000 \\ \mathrm{Al} & -2.902410000 \\ \mathrm{Cl} & -2.999530000 \\ \mathrm{Cl} & -4.703172000 \\ \mathrm{Cl} & -1.934696000 \\ & \end{array}$

1.459340000

1. 364878000

2. 892051000

3. 611671000

3. 265496000

2.123846000

1. 381627000

3.123863000

1. 851861000

1. 144220000

$-2.074873000$

$-2.345620000$

$-3.009803000$

$-1.658293000$

$-0.345758000$

0.246200000

$-1.229296000$

0.265877000

$-1.698598000$

$-2.222112000$

$-2.970756000$

$-1.409031000$

$-2.705148000$

$-2.876015000$

$-3.367927000$

$-2.545684000$

$-3.647078000$

$-0.664912000$

0.181305000

$-0.256121000$

$-1.130994000$

$-1.184123000$

$-2.759893000$

$-0.068919000$

$-1.697232000$

$-0.202357000$

$-0.353570000$

0.768471000

$-1.629981000$

$-2.452366000$

0.445755000

1. 228892000

$-0.870473000$

$-1.141789000$

$-1.917247000$

$-2.962702000$

$-1.741975000$

$-2.660924000$

$-1.579266000$

$-1.911712000$

1.281633000

1. 339774000

1.155043000

2. 232913000

$-0.124636000$

$-1.372897000$

$-2.308465000$

$-1.432312000$

$-1.343139000$

$-0.092049000$

$-0.041649000$
0.985632000

1. 829312000

0.765637000

1.464999000

$-0.635497000$

$-2.501543000$

$-3.122152000$

$-2.936052000$

$-2.431107000$

$-0.288454000$

$-0.975698000$

0.009404000

$-1.463343000$

$-1.575712000$

$-2.612163000$

$-3.073355000$

$-3.246283000$

$-2.614755000$

0.043439000

1. 417327000

1. 327960000

2.065203000

1. 947751000

$-0.799243000$

$-0.280869000$

$-1.783521000$

$-0.970486000$

0.247808000

0.870023000

$-0.707681000$

0.758642000

0.234385000

$-1.188710000$

0.515860000

2. 081406000

0.760723000

0.326932000

0.013110000

0.219053000

0.453310000

$-0.399619000$

$-0.645959000$

$-0.469611000$

$-0.764443000$

$-0.159514000$

$-0.214226000$

1. 741874000

1. 138357000

2. 173322000

2. 576981000

1.869006000

2.658395000

2. 354748000

1. 322880000

$-0.876030000$

$-1.728295000$

$-1.210386000$

$-2.003970000$

$-2.667343000$

$-0.525207000$

$-1.448375000$ 


$\begin{array}{lr}\mathrm{H} & -3.652886000 \\ \mathrm{H} & -3.710485000 \\ \mathrm{C} & -1.526602000 \\ \mathrm{H} & -0.464021000 \\ \mathrm{H} & -1.726596000 \\ \mathrm{H} & -2.124291000 \\ \mathrm{C} & 1.290352000 \\ \mathrm{C} & 1.024985000 \\ \mathrm{H} & 0.778175000\end{array}$

0.788163000

$-0.992270000$

1. 135944000

1.150907000

2.061933000

1.172106000

2.238512000

3.428691000

4.474831000

$$
\begin{array}{r}
0.085088000 \\
0.025172000 \\
-1.684180000 \\
-1.971745000 \\
-1.122977000 \\
-2.613117000 \\
0.071129000 \\
0.073101000 \\
0.079844000
\end{array}
$$

RC-1c-endo: $E=-2860.701141$

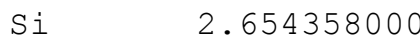

$-2.909909000$

O $\quad-1.151058000$

$\mathrm{N} \quad 1.722535000$

N $\quad-2.262342000$

B $\quad 2.063659000$

C $\quad 0.647319000$

$\mathrm{H} \quad 0.375862000$

C $\quad 1.227653000$

$\mathrm{H} \quad 1.421651000$

C $\quad 0.198787000$

$\mathrm{H} \quad-0.412718000$

C $\quad-0.110117000$

$\mathrm{H} \quad-0.946012000$

C $\quad-0.960074000$

$\mathrm{H} \quad-0.664073000$

C $\quad-0.412875000$

$\mathrm{H} \quad 0.445138000$

C $\quad-1.239218000$

C $\quad-3.272813000$

$\mathrm{H} \quad-3.197655000$

$\mathrm{H} \quad-3.099727000$

$\mathrm{H} \quad-4.272759000$

C $\quad-2.120568000$

C $\quad 1.513765000$

$\mathrm{H} \quad 1.265486000$

$\mathrm{H} \quad 2.023386000$

$\mathrm{H} \quad 0.566838000$

C $\quad 3.090418000$

$\mathrm{H} \quad 2.213609000$

$\mathrm{H} \quad 3.378018000$

$\mathrm{H} \quad 3.911787000$

C $\quad 4.180043000$

C $\quad 3.714555000$

$\mathrm{H} \quad 3.052845000$

$\mathrm{H} \quad 3.176428000$

$\mathrm{H} \quad 4.586566000$

C $\quad 4.918116000$

$\mathrm{H} \quad 5.805951000$

$\mathrm{H} \quad 5.272047000$

$\mathrm{H} \quad 4.284530000$

C $\quad 5.141470000$

$\mathrm{H} \quad 4.672646000$

$\mathrm{H} \quad 5.498264000$

$\mathrm{H} \quad 6.027216000$

Al $\quad-3.144842000$

$\mathrm{Cl} \quad-2.172491000$

$\mathrm{Cl} \quad-5.256511000$

Cl $\quad-2.163269000$

C $\quad 3.193689000$

C $\quad 4.047382000$

$\mathrm{H} \quad 4.808116000$
$-1.035149000$

0.339587000

4.503999000

0.285463000

2.522800000

1.702829000

$-0.117671000$

$-1.173011000$

2.629622000

3.704533000

2.101485000

2.736364000

0.723228000

0.286597000

1.119202000

0.176136000

2.342531000

2.656958000

3. 311673000

3.034540000

4.128987000

2.656440000

2.724420000

1.233581000

$-2.521742000$

$-3.016274000$

$-3.265225000$

$-2.261516000$

$-0.450437000$

0.014092000

$-1.327076000$

0.276372000

$-1.474667000$

$-1.909145000$

$-2.789693000$

$-1.101058000$

$-2.178745000$

$-2.644596000$

$-2.931427000$

$-2.374108000$

$-3.541601000$

$-0.279245000$

0.573848000

0.081688000

$-0.574822000$

$-1.496907000$

$-2.091944000$

$-1.736257000$

$-2.252035000$

2. 292863000

2.867603000

3.360380000
$-0.534149000$

0.021049000

$-0.498859000$

0.352237000

0.008465000

0.344121000

1.095405000

1.066078000

1.223143000

1.276476000

1. 976241000

2.627315000

1.888733000

2.436825000

$-1.275728000$

$-1.726023000$

$-1.398628000$

$-1.988904000$

$-0.607002000$

0.916631000

0.911862000

1. 935339000

0.583348000

$-0.365055000$

$-0.705510000$

0.245293000

$-1.339526000$

$-1.203678000$

$-2.263553000$

$-2.743357000$

$-2.866879000$

$-2.280937000$

0.535895000

1.940282000

1.908109000

2. 462131000

2.562406000

$-0.149712000$

0.441887000

$-1.158474000$

$-0.241944000$

0.670338000

1.183976000

$-0.306882000$

1.261359000

$-0.085177000$

$-1.907557000$

$-0.101841000$

1.664134000

$-0.519283000$

$-1.172686000$

$-1.751011000$ 
TS-1c-endo: $E=-2860.674349$

\begin{tabular}{|c|c|c|c|}
\hline $\mathrm{Si}$ & 2.679646000 & -1.104577000 & -0.427454000 \\
\hline 0 & -3.062801000 & 0.227113000 & -0.140057000 \\
\hline O & -1.587275000 & 4.541096000 & -0.114286000 \\
\hline $\mathrm{N}$ & 1.689791000 & 0.340884000 & 0.179310000 \\
\hline $\mathrm{N}$ & -2.513483000 & 2.432973000 & 0.147998000 \\
\hline B & 1.923560000 & 1.731519000 & -0.150800000 \\
\hline $\mathrm{C}$ & 0.623164000 & 0.084098000 & 0.990300000 \\
\hline $\mathrm{H}$ & 0.311587000 & -0.955641000 & 1.096072000 \\
\hline $\mathrm{C}$ & 0.866277000 & 2.742272000 & 0.401898000 \\
\hline $\mathrm{H}$ & 1.090469000 & 3.808335000 & 0.294051000 \\
\hline C & 0.254966000 & 2.364063000 & 1.664537000 \\
\hline $\mathrm{H}$ & -0.162061000 & 3.119132000 & 2.335757000 \\
\hline $\mathrm{C}$ & 0.041686000 & 1.026002000 & 1.872931000 \\
\hline $\mathrm{H}$ & -0.613018000 & 0.640803000 & 2.656840000 \\
\hline $\mathrm{C}$ & -0.948414000 & 1.160947000 & -0.933474000 \\
\hline $\mathrm{H}$ & -0.571299000 & 0.326349000 & -1.514378000 \\
\hline C & -0.394603000 & 2.498727000 & -0.830022000 \\
\hline $\mathrm{H}$ & 0.074896000 & 2.942293000 & -1.715208000 \\
\hline $\mathrm{C}$ & -1.537028000 & 3.343441000 & -0.254510000 \\
\hline C & -3.724088000 & 2.777283000 & 0.868948000 \\
\hline $\mathrm{H}$ & -3.723472000 & 3.864237000 & 1.018750000 \\
\hline $\mathrm{H}$ & -3.751126000 & 2.264789000 & 1.842058000 \\
\hline $\mathrm{H}$ & -4.610912000 & 2.480601000 & 0.290725000 \\
\hline $\mathrm{C}$ & -2.207709000 & 1.160329000 & -0.317999000 \\
\hline $\mathrm{C}$ & 1.604665000 & -2.631642000 & -0.261703000 \\
\hline $\mathrm{H}$ & 1.370065000 & -2.905547000 & 0.777594000 \\
\hline $\mathrm{H}$ & 2.153863000 & -3.475713000 & -0.709702000 \\
\hline $\mathrm{H}$ & 0.655330000 & -2.525098000 & -0.808941000 \\
\hline C & 3.112940000 & -0.842515000 & -2.231209000 \\
\hline $\mathrm{H}$ & 2.222664000 & -0.533759000 & -2.802026000 \\
\hline $\mathrm{H}$ & 3.462002000 & -1.801371000 & -2.647817000 \\
\hline $\mathrm{H}$ & 3.896482000 & -0.088553000 & -2.380132000 \\
\hline $\mathrm{C}$ & 4.204145000 & -1.211471000 & 0.724171000 \\
\hline C & 3.736290000 & -1.403213000 & 2.180878000 \\
\hline $\mathrm{H}$ & 3.143190000 & -2.322505000 & 2.312476000 \\
\hline $\mathrm{H}$ & 3.128320000 & -0.553879000 & 2.534042000 \\
\hline $\mathrm{H}$ & 4.609675000 & -1.480231000 & 2.852535000 \\
\hline C & 5.039707000 & -2.434240000 & 0.285272000 \\
\hline $\mathrm{H}$ & 5.926346000 & -2.538519000 & 0.935608000 \\
\hline $\mathrm{H}$ & 5.404409000 & -2.335200000 & -0.750619000 \\
\hline $\mathrm{H}$ & 4.470704000 & -3.375490000 & 0.356529000 \\
\hline $\mathrm{C}$ & 5.074374000 & 0.055788000 & 0.634261000 \\
\hline $\mathrm{H}$ & 4.538204000 & 0.953047000 & 0.981101000 \\
\hline $\mathrm{H}$ & 5.418576000 & 0.253860000 & -0.392753000 \\
\hline $\mathrm{H}$ & 5.970010000 & -0.057578000 & 1.270920000 \\
\hline Al & -2.989278000 & -1.588105000 & -0.164462000 \\
\hline $\mathrm{Cl}$ & -1.818436000 & -2.147952000 & -1.889551000 \\
\hline $\mathrm{Cl}$ & -5.009716000 & -2.256353000 & -0.249509000 \\
\hline $\mathrm{Cl}$ & -1.986913000 & -2.119860000 & 1.675667000 \\
\hline C & 3.012197000 & 2.229168000 & -1.096154000 \\
\hline C & 3.835517000 & 2.735753000 & -1.838322000 \\
\hline $\mathrm{H}$ & 4.570913000 & 3.166891000 & -2.494652000 \\
\hline
\end{tabular}

2c-endo: $E=-2860.707568$

$\begin{array}{lrr}\text { Si } & -2.591323000 & -1.055380000 \\ \mathrm{O} & 2.760312000 & 0.273864000 \\ \mathrm{O} & 1.461204000 & 4.608937000 \\ \mathrm{~N} & -1.733865000 & 0.461864000 \\ \mathrm{~N} & 2.364299000 & 2.496525000 \\ \mathrm{~B} & -2.200123000 & 1.792873000\end{array}$

0.677341000

$-0.236620000$

0.101941000

0.198453000

$-0.161807000$

0.206265000 


\begin{tabular}{|c|c|c|c|}
\hline C & -0.374804000 & 0.347639000 & -0.395684000 \\
\hline $\mathrm{H}$ & -0.062834000 & -0.697264000 & -0.458240000 \\
\hline $\mathrm{C}$ & -1.095902000 & 2.828768000 & -0.348232000 \\
\hline $\mathrm{H}$ & -1.395854000 & 3.882582000 & -0.341604000 \\
\hline $\mathrm{C}$ & -0.692188000 & 2.319790000 & -1.714672000 \\
\hline $\mathrm{H}$ & -0.716047000 & 2.969098000 & -2.593314000 \\
\hline $\mathrm{C}$ & -0.309889000 & 1.034268000 & -1.744962000 \\
\hline $\mathrm{H}$ & 0.048635000 & 0.501176000 & -2.627141000 \\
\hline $\mathrm{C}$ & 0.585569000 & 1.140501000 & 0.560570000 \\
\hline $\mathrm{H}$ & 0.616876000 & 0.635256000 & 1.537527000 \\
\hline $\mathrm{C}$ & 0.123275000 & 2.610846000 & 0.623653000 \\
\hline $\mathrm{H}$ & -0.149867000 & 2.929344000 & 1.639916000 \\
\hline C & 1.330528000 & 3.419704000 & 0.186390000 \\
\hline C & 3.660549000 & 2.901060000 & -0.689689000 \\
\hline $\mathrm{H}$ & 3.790762000 & 3.968584000 & -0.476228000 \\
\hline $\mathrm{H}$ & 3.690464000 & 2.734209000 & -1.776438000 \\
\hline $\mathrm{H}$ & 4.450959000 & 2.307321000 & -0.212566000 \\
\hline $\mathrm{C}$ & 1.989393000 & 1.224061000 & 0.028734000 \\
\hline C & -1.309229000 & -2.287058000 & 1.289720000 \\
\hline $\mathrm{H}$ & -0.649078000 & -2.674619000 & 0.499725000 \\
\hline $\mathrm{H}$ & -1.836279000 & -3.146773000 & 1.734782000 \\
\hline $\mathrm{H}$ & -0.665951000 & -1.850648000 & 2.069232000 \\
\hline $\mathrm{C}$ & -3.791277000 & -0.688965000 & 2.074433000 \\
\hline $\mathrm{H}$ & -3.271536000 & -0.196371000 & 2.911795000 \\
\hline $\mathrm{H}$ & -4.204378000 & -1.641222000 & 2.445712000 \\
\hline $\mathrm{H}$ & -4.622691000 & -0.042584000 & 1.764136000 \\
\hline $\mathrm{C}$ & -3.491398000 & -1.735119000 & -0.872721000 \\
\hline C & -2.483856000 & -1.915071000 & -2.025978000 \\
\hline $\mathrm{H}$ & -1.641348000 & -2.572171000 & -1.755501000 \\
\hline $\mathrm{H}$ & -2.066512000 & -0.949938000 & -2.353532000 \\
\hline $\mathrm{H}$ & -2.983567000 & -2.366217000 & -2.902313000 \\
\hline $\mathrm{C}$ & -4.122634000 & -3.098901000 & -0.525500000 \\
\hline $\mathrm{H}$ & -4.669541000 & -3.500511000 & -1.397900000 \\
\hline $\mathrm{H}$ & -4.844798000 & -3.022952000 & 0.304703000 \\
\hline $\mathrm{H}$ & -3.362566000 & -3.846286000 & -0.245058000 \\
\hline C & -4.594673000 & -0.755561000 & -1.317660000 \\
\hline $\mathrm{H}$ & -4.188942000 & 0.242239000 & -1.550852000 \\
\hline $\mathrm{H}$ & -5.372036000 & -0.625906000 & -0.547392000 \\
\hline $\mathrm{H}$ & -5.093414000 & -1.131186000 & -2.229597000 \\
\hline Al & 2.944197000 & -1.538507000 & 0.133561000 \\
\hline $\mathrm{Cl}$ & 2.239658000 & -1.666247000 & 2.155753000 \\
\hline $\mathrm{Cl}$ & 5.023934000 & -1.892460000 & -0.117341000 \\
\hline $\mathrm{Cl}$ & 1.688739000 & -2.531627000 & -1.294243000 \\
\hline $\mathrm{C}$ & -3.564996000 & 2.288195000 & 0.690045000 \\
\hline C & -4.625716000 & 2.778425000 & 1.036953000 \\
\hline $\mathrm{H}$ & -5.567182000 & 3.195426000 & 1.347410000 \\
\hline
\end{tabular}

RC-1c-exo: $E=-2860.699051$

$\begin{array}{lrrr}\mathrm{Si} & -2.190269000 & 0.923726000 & -0.701534000 \\ \mathrm{O} & 2.300465000 & -0.388963000 & -0.875495000 \\ \mathrm{O} & -1.132717000 & -3.216030000 & -1.832925000 \\ \mathrm{~N} & -1.517876000 & -0.113255000 & 0.662073000 \\ \mathrm{~N} & 0.570780000 & -1.644366000 & -1.672698000 \\ \mathrm{~B} & -2.205663000 & -1.264827000 & 1.239876000 \\ \mathrm{C} & -0.394321000 & 0.359341000 & 1.286942000 \\ \mathrm{H} & 0.119793000 & 1.208726000 & 0.834633000 \\ \mathrm{C} & -1.658092000 & -1.817486000 & 2.551742000 \\ \mathrm{H} & -2.133665000 & -2.658846000 & 3.064942000 \\ \mathrm{C} & -0.542376000 & -1.239309000 & 3.115447000 \\ \mathrm{H} & -0.123842000 & -1.604598000 & 4.059800000 \\ \mathrm{C} & 0.112274000 & -0.164210000 & 2.460577000 \\ \mathrm{H} & 1.023756000 & 0.274051000 & 2.869804000 \\ \mathrm{C} & 1.257222000 & -2.174192000 & 0.466823000\end{array}$




$\begin{array}{lrrr}\mathrm{H} & 1.886805000 & -2.103481000 & 1.348436000 \\ \mathrm{C} & 0.223216000 & -2.994765000 & 0.199521000 \\ \mathrm{H} & -0.209031000 & -3.778192000 & 0.816267000 \\ \mathrm{C} & -0.259872000 & -2.705515000 & -1.184498000 \\ \mathrm{C} & 0.515730000 & -1.132197000 & -3.032947000 \\ \mathrm{H} & -0.410390000 & -0.567064000 & -3.198097000 \\ \mathrm{H} & 0.550475000 & -1.973530000 & -3.738724000 \\ \mathrm{H} & 1.379609000 & -0.475554000 & -3.190415000 \\ \mathrm{C} & 1.458307000 & -1.301472000 & -0.714092000 \\ \mathrm{C} & -0.747565000 & 1.873260000 & -1.457324000 \\ \mathrm{H} & -0.428887000 & 2.731040000 & -0.848157000 \\ \mathrm{H} & -1.064081000 & 2.261637000 & -2.438534000 \\ \mathrm{H} & 0.145376000 & 1.252224000 & -1.614957000 \\ \mathrm{C} & -2.996183000 & -0.136659000 & -2.026631000 \\ \mathrm{H} & -2.482615000 & -1.097737000 & -2.171549000 \\ \mathrm{H} & -2.972612000 & 0.420148000 & -2.977976000 \\ \mathrm{H} & -4.041730000 & -0.366550000 & -1.783430000 \\ \mathrm{C} & -3.427838000 & 2.144241000 & 0.103103000 \\ \mathrm{C} & -2.720482000 & 2.971570000 & 1.194897000 \\ \mathrm{H} & -1.870717000 & 3.551517000 & 0.800420000 \\ \mathrm{H} & -2.344293000 & 2.337227000 & 2.013769000 \\ \mathrm{H} & -3.428412000 & 3.693582000 & 1.639529000 \\ \mathrm{C} & -3.943024000 & 3.085676000 & -1.007017000 \\ \mathrm{H} & -4.659176000 & 3.814017000 & -0.586201000 \\ \mathrm{H} & -4.468512000 & 2.535080000 & -1.804968000 \\ \mathrm{H} & -3.127449000 & 3.662526000 & -1.473165000 \\ \mathrm{C} & -4.616543000 & 1.395122000 & 0.733770000 \\ \mathrm{H} & -4.296617000 & 0.721358000 & 1.544035000 \\ \mathrm{H} & -5.167098000 & 0.787502000 & -0.000826000 \\ \mathrm{H} & -5.329206000 & 2.118710000 & 1.168739000 \\ \mathrm{~A} 1 & 3.509993000 & 0.663660000 & 0.062186000 \\ \mathrm{C} 1 & 2.457269000 & 2.515782000 & 0.279197000 \\ \mathrm{Cl} & 5.205849000 & 0.729429000 & -1.217270000 \\ \mathrm{Cl} & 3.837083000 & -0.320372000 & 1.941630000 \\ \mathrm{C} & -3.437057000 & -1.910552000 & 0.582978000 \\ \mathrm{C} & -4.399093000 & -2.508320000 & 0.133356000 \\ \mathrm{H} & -5.246863000 & -3.025557000 & -0.278314000\end{array}$

TS-1c-exo: $E=-2860.669949$

$\begin{array}{lrrr}\mathrm{Si} & 1.840062000 & -1.276636000 & -0.631788000 \\ \mathrm{O} & -2.011555000 & 0.337223000 & -0.753798000 \\ \mathrm{O} & 0.618463000 & 4.028833000 & -1.114041000 \\ \mathrm{~N} & 1.535642000 & 0.127025000 & 0.539346000 \\ \mathrm{~N} & -0.529009000 & 2.018104000 & -1.226182000 \\ \mathrm{~B} & 2.119088000 & 1.451625000 & 0.471951000 \\ \mathrm{C} & 0.747858000 & -0.137850000 & 1.620774000 \\ \mathrm{H} & 0.167862000 & -1.060919000 & 1.603877000 \\ \mathrm{C} & 1.626894000 & 2.447527000 & 1.564947000 \\ \mathrm{H} & 2.077309000 & 3.443957000 & 1.579125000 \\ \mathrm{C} & 1.340767000 & 1.847811000 & 2.844051000 \\ \mathrm{H} & 1.391084000 & 2.432482000 & 3.767160000 \\ \mathrm{C} & 0.813346000 & 0.580083000 & 2.839123000 \\ \mathrm{H} & 0.360731000 & 0.127719000 & 3.723237000 \\ \mathrm{C} & -1.058225000 & 1.758816000 & 0.988501000 \\ \mathrm{H} & -1.636203000 & 1.426596000 & 1.843766000 \\ \mathrm{C} & -0.130012000 & 2.846452000 & 0.917931000 \\ \mathrm{H} & -0.273967000 & 3.741393000 & 1.526919000 \\ \mathrm{C} & 0.067508000 & 3.107031000 & -0.569034000 \\ \mathrm{C} & -0.571062000 & 1.867750000 & -2.668191000 \\ \mathrm{H} & 0.357217000 & 2.279202000 & -3.084403000 \\ \mathrm{H} & -1.428305000 & 2.411053000 & -3.096461000 \\ \mathrm{H} & -0.664144000 & 0.804194000 & -2.919299000 \\ \mathrm{C} & -1.276904000 & 1.282958000 & -0.323292000\end{array}$




$\begin{array}{lrrr}\mathrm{C} & 0.245958000 & -2.250515000 & -0.777650000 \\ \mathrm{H} & -0.183815000 & -2.598579000 & 0.172177000 \\ \mathrm{H} & 0.444445000 & -3.142169000 & -1.394404000 \\ \mathrm{H} & -0.524093000 & -1.661036000 & -1.296208000 \\ \mathrm{C} & 2.314087000 & -0.638543000 & -2.327445000 \\ \mathrm{H} & 1.674761000 & 0.198206000 & -2.640230000 \\ \mathrm{H} & 2.168948000 & -1.463583000 & -3.044141000 \\ \mathrm{H} & 3.358174000 & -0.304907000 & -2.380885000 \\ \mathrm{C} & 3.248934000 & -2.290153000 & 0.171618000 \\ \mathrm{C} & 2.769941000 & -2.923199000 & 1.492547000 \\ \mathrm{H} & 1.899089000 & -3.582856000 & 1.347961000 \\ \mathrm{H} & 2.498988000 & -2.163020000 & 2.243679000 \\ \mathrm{H} & 3.576523000 & -3.535423000 & 1.933846000 \\ \mathrm{C} & 3.654240000 & -3.407581000 & -0.814182000 \\ \mathrm{H} & 4.465974000 & -4.018351000 & -0.380681000 \\ \mathrm{H} & 4.024518000 & -3.002145000 & -1.769945000 \\ \mathrm{H} & 2.817129000 & -4.089762000 & -1.036598000 \\ \mathrm{C} & 4.465768000 & -1.386087000 & 0.450444000 \\ \mathrm{H} & 4.231310000 & -0.592830000 & 1.178613000 \\ \mathrm{H} & 4.842462000 & -0.898521000 & -0.462639000 \\ \mathrm{H} & 5.293234000 & -1.984206000 & 0.872060000 \\ \mathrm{Al} & -3.284076000 & -0.768962000 & -0.036773000 \\ \mathrm{Cl} & -3.443263000 & -2.354438000 & -1.457731000 \\ \mathrm{Cl} & -5.031643000 & 0.439909000 & 0.184700000 \\ \mathrm{Cl} & -2.501062000 & -1.428657000 & 1.867542000 \\ \mathrm{C} & 3.100105000 & 1.909035000 & -0.602356000 \\ \mathrm{C} & 3.890918000 & 2.378472000 & -1.401717000 \\ \mathrm{H} & 4.586486000 & 2.782538000 & -2.115719000\end{array}$

\section{2c-exo: $E=-2860.704628$}

$\begin{array}{lr}\text { Si } & 1.809028000 \\ \mathrm{O} & -1.957773000 \\ \mathrm{O} & 0.288798000 \\ \mathrm{~N} & 1.497438000 \\ \mathrm{~N} & -0.900702000 \\ \mathrm{~B} & 2.222834000 \\ \mathrm{C} & 0.531230000 \\ \mathrm{H} & 0.022808000 \\ \mathrm{C} & 1.735692000 \\ \mathrm{H} & 2.248615000 \\ \mathrm{C} & 1.845594000 \\ \mathrm{H} & 2.403636000 \\ \mathrm{C} & 1.214147000 \\ \mathrm{H} & 1.148272000 \\ \mathrm{C} & -0.531933000 \\ \mathrm{H} & -1.292948000 \\ \mathrm{C} & 0.199442000 \\ \mathrm{H} & -0.209428000 \\ \mathrm{C} & -0.078266000 \\ \mathrm{C} & -1.377153000 \\ \mathrm{H} & -0.917547000 \\ \mathrm{H} & -1.087252000 \\ \mathrm{H} & -2.469083000 \\ \mathrm{C} & -1.208580000 \\ \mathrm{C} & 0.207469000 \\ \mathrm{H} & -0.262945000 \\ \mathrm{H} & 0.420312000 \\ \mathrm{H} & -0.534986000 \\ \mathrm{C} & 2.370446000 \\ \mathrm{H} & 1.698730000 \\ \mathrm{H} & 2.331282000 \\ \mathrm{H} & 3.391712000 \\ \mathrm{C} & 3.144997000 \\ & \end{array}$

$$
\begin{array}{rr}
-1.264087000 & -0.767230000 \\
0.297195000 & -0.620383000 \\
4.238804000 & -0.783318000 \\
0.221797000 & 0.215581000 \\
2.262278000 & -0.975736000 \\
1.435301000 & 0.204094000 \\
0.158640000 & 1.348679000 \\
-0.807526000 & 1.381582000 \\
2.445640000 & 1.359870000 \\
3.413486000 & 1.375231000 \\
1.673355000 & 2.657348000 \\
2.071169000 & 3.508327000 \\
0.491591000 & 2.661261000 \\
-0.184559000 & 3.515152000 \\
1.295344000 & 1.132942000 \\
1.212591000 & 1.921458000 \\
2.653263000 & 1.096248000 \\
3.362196000 & 1.832092000 \\
3.210728000 & -0.287846000 \\
2.443434000 & -2.340597000 \\
1.692251000 & -2.998707000 \\
3.450568000 & -2.662439000 \\
2.327865000 & -2.367131000 \\
1.209901000 & -0.205877000 \\
-2.236926000 & -0.923716000 \\
-2.501543000 & 0.034748000 \\
-3.180111000 & -1.452804000 \\
-1.690542000 & -1.523891000 \\
-0.769503000 & -2.490664000 \\
-0.006400000 & -2.915453000 \\
-1.657155000 & -3.142966000 \\
-0.366289000 & -2.507179000 \\
-2.297167000 & 0.141612000 \\
& \\
-1.25 &
\end{array}
$$




$\begin{array}{lr}\mathrm{C} & 2.577551000 \\ \mathrm{H} & 1.695759000 \\ \mathrm{H} & 2.288688000 \\ \mathrm{H} & 3.339138000 \\ \mathrm{C} & 3.557259000 \\ \mathrm{H} & 4.316105000 \\ \mathrm{H} & 3.998281000 \\ \mathrm{H} & 2.705813000 \\ \mathrm{C} & 4.381453000 \\ \mathrm{H} & 4.143158000 \\ \mathrm{H} & 4.816245000 \\ \mathrm{H} & 5.166713000 \\ \mathrm{Al} & -3.269401000 \\ \mathrm{Cl} & -3.500375000 \\ \mathrm{Cl} & -4.901460000 \\ \mathrm{Cl} & -2.509356000 \\ \mathrm{C} & 3.312676000 \\ \mathrm{C} & 4.186175000 \\ \mathrm{H} & 4.956276000\end{array}$

1d: B

C $\mathrm{H}$

$$
\begin{array}{r}
-2.840659000 \\
-3.483009000 \\
-2.028605000 \\
-3.448651000 \\
-3.479367000 \\
-4.100608000 \\
-3.139079000 \\
-4.138121000 \\
-1.427502000 \\
-0.596298000 \\
-0.992417000 \\
-2.035739000 \\
-0.847161000 \\
-2.312513000 \\
0.508784000 \\
-1.597303000 \\
1.844198000 \\
2.269759000 \\
2.632968000
\end{array}
$$

$$
\begin{gathered}
E=-502.924826 \\
-0.132732000 \\
-1.154753000 \\
-0.503378000 \\
0.308337000 \\
-0.796423000 \\
-0.061985000 \\
-1.700502000 \\
-0.321350000 \\
-2.595871000 \\
-3.436585000 \\
-2.867307000 \\
-3.896750000 \\
-1.805450000 \\
-2.022586000 \\
1.253259000 \\
1.698884000 \\
2.019979000 \\
3.433984000 \\
3.734410000 \\
3.894375000 \\
3.741738000
\end{gathered}
$$

1.467997000

1.312416000

2. 154270000

1.989061000

$-0.760204000$

$-0.251005000$

$-1.711392000$

$-0.998828000$

0.439336000

1.121965000

$-0.474667000$

0.923786000

0.037717000

$-1.484073000$

0.288245000

1. 901129000

$-0.787919000$

$-1.523774000$

$-2.180796000$

-0.045685000
0.037306000
-0.083990000
-0.143559000
0.109533000
0.900801000
0.284153000
-0.824846000
0.066061000
0.128531000
0.018597000
0.039020000
-0.058457000
-0.101821000
-0.079314000
-0.295828000
0.163652000
0.121893000
0.880945000
0.330095000
-0.868581000

RC-1d-endo: $E=-2525.177063$

$\begin{array}{rrrr}\mathrm{O} & -1.627268000 & 1.244642000 & -0.267869000 \\ \mathrm{O} & 1.993514000 & 3.949985000 & -0.793298000 \\ \mathrm{~N} & 2.004405000 & -1.089676000 & 0.530490000 \\ \mathrm{~N} & 0.012907000 & 2.824226000 & -0.315604000 \\ \mathrm{~B} & 3.181852000 & -0.226117000 & 0.344632000 \\ \mathrm{C} & 0.935925000 & -0.698656000 & 1.313758000 \\ \mathrm{H} & 0.106045000 & -1.398275000 & 1.394013000 \\ \mathrm{C} & 4.408106000 & -0.656498000 & -0.547047000 \\ \mathrm{H} & 4.726088000 & -1.695181000 & -0.359797000 \\ \mathrm{H} & 5.270199000 & 0.010017000 & -0.388294000 \\ \mathrm{H} & 4.140014000 & -0.626963000 & -1.616724000 \\ \mathrm{C} & 3.097840000 & 1.108597000 & 1.090347000 \\ \mathrm{H} & 3.908618000 & 1.841886000 & 1.030392000 \\ \mathrm{C} & 1.997727000 & 1.416651000 & 1.857022000 \\ \mathrm{H} & 1.930761000 & 2.369293000 & 2.394643000 \\ \mathrm{C} & 0.903534000 & 0.509364000 & 1.958003000 \\ \mathrm{H} & 0.013473000 & 0.748481000 & 2.541663000 \\ \mathrm{C} & 0.491246000 & 0.890134000 & -1.481529000\end{array}$




$\begin{array}{lr}\mathrm{H} & 0.306827000 \\ \mathrm{C} & 1.574107000 \\ \mathrm{H} & 2.494153000 \\ \mathrm{C} & 1.316040000 \\ \mathrm{C} & -0.633170000 \\ \mathrm{H} & -0.767056000 \\ \mathrm{H} & -1.614564000 \\ \mathrm{H} & 0.016607000 \\ \mathrm{C} & -0.489068000 \\ \mathrm{Al} & -2.632976000 \\ \mathrm{Cl} & -1.563953000 \\ \mathrm{Cl} & -4.521413000 \\ \mathrm{Cl} & -2.599231000 \\ \mathrm{C} & 1.874945000 \\ \mathrm{O} & 2.333864000 \\ \mathrm{O} & 1.183725000 \\ \mathrm{C} & 0.849265000 \\ \mathrm{H} & 1.760727000 \\ \mathrm{H} & 0.305146000 \\ \mathrm{H} & 0.209699000\end{array}$

$$
\begin{array}{r}
-0.107641000 \\
1.674832000 \\
1.488600000 \\
2.964416000 \\
3.810304000 \\
3.408142000 \\
4.082973000 \\
4.693549000 \\
1.613709000 \\
-0.297474000 \\
-1.858794000 \\
0.172036000 \\
-0.580698000 \\
-2.327828000 \\
-2.521952000 \\
-3.216126000 \\
-4.442401000 \\
-4.961824000 \\
-5.043311000 \\
-4.235251000
\end{array}
$$

\begin{tabular}{|c|c|}
\hline 0 & -1.817721000 \\
\hline 0 & 1.589067000 \\
\hline $\mathrm{N}$ & 2.157695000 \\
\hline $\mathrm{N}$ & -0.282880000 \\
\hline B & 3.181568000 \\
\hline $\mathrm{C}$ & 1.042677000 \\
\hline $\mathrm{H}$ & 0.230720000 \\
\hline $\mathrm{C}$ & 4.463968000 \\
\hline $\mathrm{H}$ & 4.960775000 \\
\hline $\mathrm{H}$ & 5.182945000 \\
\hline $\mathrm{H}$ & 4.184601000 \\
\hline $\mathrm{C}$ & 2.781264000 \\
\hline $\mathrm{H}$ & 3.533347000 \\
\hline $\mathrm{C}$ & 1.950749000 \\
\hline $\mathrm{H}$ & 1.952552000 \\
\hline $\mathrm{C}$ & 1.019235000 \\
\hline $\mathrm{H}$ & 0.191523000 \\
\hline $\mathrm{C}$ & 0.536548000 \\
\hline $\mathrm{H}$ & 0.495632000 \\
\hline $\mathrm{C}$ & 1.661831000 \\
\hline $\mathrm{H}$ & 2.390349000 \\
\hline $\mathrm{C}$ & 1.052667000 \\
\hline $\mathrm{C}$ & -1.219111000 \\
\hline $\mathrm{H}$ & -1.654769000 \\
\hline $\mathrm{H}$ & -2.032102000 \\
\hline $\mathrm{H}$ & -0.666529000 \\
\hline $\mathrm{C}$ & -0.609590000 \\
\hline Al & -2.605975000 \\
\hline $\mathrm{Cl}$ & -1.462942000 \\
\hline $\mathrm{Cl}$ & -4.619062000 \\
\hline $\mathrm{Cl}$ & -2.369053000 \\
\hline $\mathrm{C}$ & 2.154928000 \\
\hline 0 & 2.829954000 \\
\hline O & 1.319174000 \\
\hline $\mathrm{C}$ & 1.065807000 \\
\hline $\mathrm{H}$ & 2.006939000 \\
\hline $\mathrm{H}$ & 0.407846000 \\
\hline $\mathrm{H}$ & 0.560661000 \\
\hline
\end{tabular}

TS-1d-endo: $E=-2525.149399$
-1.871235000
-1.610446000
-2.160340000
-0.887778000
0.532918000
1.547827000
0.120816000
0.565689000
-0.636854000
-0.048379000
-1.081309000
-0.896975000
2.071683000
-0.169779000
-1.266042000
0.539035000
-0.125536000
-0.454484000
0.611914000
-0.995400000
$-0.515295000$

$-0.532934000$

0.442425000

$-0.277894000$

0.057461000

1.127788000

1.237797000

$-0.764363000$

$-0.384708000$

$-0.779481000$

$-1.803550000$

0.442099000

0.287758000

1. 610603000

2.171967000

1. 871328000

2.569516000

$-1.082927000$

$-1.566088000$

$-0.982814000$

$-1.798729000$

$-0.600343000$

0.266239000

1.201179000

$-0.448825000$

0.456278000

$-0.629068000$

$-0.124483000$

$-1.105682000$

$-0.791860000$

2.015247000

$-0.051006000$

$-0.977493000$

0.643845000

0.132640000

0.025364000

0.864810000

$-0.841035000$

2d-endo: $E=-2525.188348$ 
1. 355373000

2.273693000

$-0.450724000$

3.344865000

0.964153000

0.229083000

4.820899000

5.228365000

5.480413000

4.848025000

2.768705000

3. 512073000

2. 077910000

2.341855000

1. 137956000

0.509680000

0.569312000

0.411371000

1. 659580000

2. 120547000

0.925133000

$-1.465753000$

$-1.758866000$

$-2.348906000$

$-1.030792000$

$-0.689430000$

$-2.697895000$

$-1.737956000$

$-4.735229000$

$-2.241379000$

2. 381084000

3.404469000

1. 202776000

1.153356000

1.754780000

0.095645000

1. 534795000
1.034041000

4.218322000

$-0.831797000$

2. 801978000

0.122544000

$-0.245607000$

$-1.038586000$

$-0.211117000$

$-0.999018000$

0.669098000

$-0.652985000$

1. 597754000

2.399022000

1. 559808000

2. 264223000

0.611194000

0.448843000

0.699119000

0.082349000

1. 780137000

1.770800000

3.099826000

3. 820131000

3. 815402000

3. 610296000

4.791857000

1. 482321000

$-0.614056000$

$-1.834247000$

$-0.158400000$

$-1.202012000$

$-2.194030000$

$-2.739348000$

$-2.817386000$

$-4.199993000$

$-4.801570000$

$-4.481745000$

$-4.352325000$
$-0.139332000$

$-0.647709000$

0.188415000

$-0.361263000$

0.073968000

0.584446000

0.719615000

$-0.317837000$

0.336283000

$-0.292650000$

$-1.329063000$

0.343409000

0.256945000

1. 690904000

2.483545000

1. 817734000

2.694986000

$-0.601119000$

$-1.498361000$

$-0.758249000$

$-1.757060000$

$-0.602998000$

$-0.123003000$

0.936865000

$-0.740618000$

$-0.384752000$

$-0.345644000$

0.012468000

$-1.467113000$

$-0.376541000$

2. 019114000

$-0.112253000$

$-0.455328000$

0.012222000

$-0.341932000$

0.356299000

$-0.283961000$

$-1.362477000$
N-Methylmaleimide:

$\begin{array}{lr}\mathrm{O} & -2.287560000 \\ \mathrm{O} & 2.285731000 \\ \mathrm{~N} & -0.009715000 \\ \mathrm{C} & -0.641106000 \\ \mathrm{H} & -1.319173000 \\ \mathrm{C} & 0.697415000 \\ \mathrm{H} & 1.404891000 \\ \mathrm{C} & 1.153260000 \\ \mathrm{C} & -0.043851000 \\ \mathrm{H} & 0.992970000 \\ \mathrm{H} & -0.562759000 \\ \mathrm{H} & -0.566111000 \\ \mathrm{C} & -1.143582000\end{array}$

$E=-2022.232321$

0.171919000

0.241760000

0.591207000

$-1.640547000$

$-2.493318000$

$-1.617628000$

$-2.446175000$

$-0.182880000$

2.039090000

2. 399503000

2.415578000

2. 415276000

$-0.221159000$
-0.000063000
-0.000056000
0.000055000
0.000028000
0.000032000
0.000016000
-0.000004000
0.000011000
0.000021000
-0.001912000
0.894496000
-0.892607000
0.000018000

$-0.480588000$

$-0.181890000$

$-0.204772000$

0.050498000

0.129949000

1.278715000

$-0.734231000$

$-1.633608000$ 


$\begin{array}{rrr}0.041019000 & -0.848238000 & 2.253029000 \\ -0.997751000 & -1.073252000 & 2.536943000 \\ 0.600681000 & -0.671145000 & 3.185875000 \\ 0.452911000 & -1.763938000 & 1.797017000 \\ 1.229723000 & 1.488584000 & 1.566613000 \\ 1.877784000 & 1.444856000 & 2.449219000 \\ 1.380783000 & 2.548801000 & 0.705767000 \\ 2.128639000 & 3.329556000 & 0.885243000 \\ 0.566784000 & 2.648431000 & -0.458968000 \\ 0.672068000 & 3.490452000 & -1.145924000 \\ 2.727032000 & 0.392211000 & -1.780085000 \\ 2.770615000 & 1.208649000 & -2.498457000 \\ 1.976243000 & -0.715684000 & -1.792313000 \\ 1.245687000 & -1.048515000 & -2.527263000 \\ 2.284726000 & -1.515131000 & -0.559832000 \\ 3.782588000 & -1.183486000 & 1.427764000 \\ 4.006476000 & -2.259034000 & 1.442042000 \\ 3.050134000 & -0.960917000 & 2.219404000 \\ 4.697782000 & -0.605776000 & 1.610525000 \\ 3.582239000 & 0.375779000 & -0.542654000 \\ -2.070913000 & -0.421119000 & -2.365764000 \\ -2.435925000 & 0.542013000 & -2.752212000 \\ -2.770504000 & -1.200242000 & -2.708543000 \\ -1.093443000 & -0.626653000 & -2.830312000 \\ -1.670594000 & -2.308537000 & -0.035234000 \\ -0.633974000 & -2.610230000 & -0.249738000 \\ -2.343019000 & -2.919767000 & -0.659360000 \\ -1.884275000 & -2.537593000 & 1.017265000 \\ -3.572384000 & 0.183544000 & 0.299638000 \\ -3.798100000 & 1.640120000 & -0.150360000 \\ -3.884508000 & 1.729642000 & -1.245457000 \\ -2.983624000 & 2.304386000 & 0.180421000 \\ -4.736590000 & 2.031026000 & 0.282651000 \\ -4.753147000 & -0.689607000 & -0.175625000 \\ -5.700117000 & -0.319266000 & 0.257207000 \\ -4.640919000 & -1.741194000 & 0.136301000 \\ -4.867680000 & -0.673066000 & -1.271994000 \\ -3.497537000 & 0.141645000 & 1.837414000 \\ -2.671149000 & 0.758478000 & 2.224504000 \\ -4.363424000 & -0.883973000 & 2.217597000 \\ -43460000 & 0.532031000 & 2.274532000\end{array}$

\section{TS-1a-endo' : $E=-1200.372089$}

$\begin{array}{lrr}\mathrm{Si} & 2.281435000 & -0.272312000 \\ \mathrm{O} & -2.835947000 & -2.603487000 \\ \mathrm{O} & -4.243003000 & 1.743633000 \\ \mathrm{~N} & 0.714939000 & 0.276201000 \\ \mathrm{~N} & -3.767040000 & -0.510310000 \\ \mathrm{~B} & 0.216622000 & 1.626239000 \\ \mathrm{C} & -0.143960000 & -0.722978000 \\ \mathrm{H} & 0.167778000 & -1.755289000 \\ \mathrm{C} & 0.951884000 & 2.908975000 \\ \mathrm{H} & 2.019066000 & 2.949963000 \\ \mathrm{H} & 0.480861000 & 3.832664000 \\ \mathrm{H} & 0.900666000 & 2.939062000 \\ \mathrm{C} & -1.183559000 & 1.736090000 \\ \mathrm{H} & -1.706684000 & 2.697326000 \\ \mathrm{C} & -1.499799000 & 0.791570000 \\ \mathrm{H} & -2.246218000 & 1.006892000 \\ \mathrm{C} & -0.982037000 & -0.474255000 \\ \mathrm{H} & -1.328943000 & -1.321085000 \\ \mathrm{C} & -1.665187000 & -0.545862000 \\ \mathrm{H} & -0.997508000 & -0.949133000 \\ \mathrm{C} & -2.081409000 & 0.806098000\end{array}$




$\begin{array}{lr}\mathrm{H} & -1.806993000 \\ \mathrm{C} & -3.479730000 \\ \mathrm{C} & -4.986811000 \\ \mathrm{H} & -5.159696000 \\ \mathrm{H} & -5.823516000 \\ \mathrm{H} & -4.912178000 \\ \mathrm{C} & -2.767071000 \\ \mathrm{C} & 2.025944000 \\ \mathrm{H} & 1.851012000 \\ \mathrm{H} & 2.931741000 \\ \mathrm{H} & 1.181794000 \\ \mathrm{C} & 2.816974000 \\ \mathrm{H} & 1.979636000 \\ \mathrm{H} & 3.607229000 \\ \mathrm{H} & 3.217709000 \\ \mathrm{C} & 3.581089000 \\ \mathrm{C} & 3.103762000 \\ \mathrm{H} & 2.972901000 \\ \mathrm{H} & 2.147779000 \\ \mathrm{H} & 3.846329000 \\ \mathrm{C} & 4.923368000 \\ \mathrm{H} & 5.689002000 \\ \mathrm{H} & 5.307943000 \\ \mathrm{H} & 4.843167000 \\ \mathrm{C} & 3.772846000 \\ \mathrm{H} & 2.837746000 \\ \mathrm{H} & 4.131623000 \\ \mathrm{H} & 4.523034000 \\ & \end{array}$

1.577038000
0.811535000

$-0.923889000$

$-1.984642000$

$-0.313129000$

$-0.797890000$

$-1.393322000$

$-1.998899000$

$-2.776717000$

$-2.282063000$

$-2.016407000$

0.844145000

1. 033608000

0.324630000

1. 812065000

$-0.314066000$

$-1.265574000$

$-2.298214000$

$-0.936554000$

$-1.299092000$

$-0.817840000$

$-0.848839000$

$-0.159397000$

$-1.836638000$

1.095584000

1.489154000

1. 817831000

1.069744000
-1.672437000
-0.411075000
0.594991000
0.369807000
0.228494000
1.687773000
-0.499600000
-1.428412000
-0.670127000
-1.988318000
-2.136480000
-2.126279000
-2.816187000
-2.692611000
-1.797180000
0.695826000
1.810814000
1.448321000
2.249296000
2.628367000
0.126123000
0.922319000
-0.670409000
-0.287869000
1.288102000
1.717894000
0.536445000
2.098970000

$-0.756731000$

$-0.229188000$

$-0.097836000$

$-0.236022000$

$-0.109030000$

$-0.132238000$

0.239489000

0.211897000

$-0.478555000$

$-0.725033000$

0.373922000

$-1.325360000$

0.394412000

0.478079000

1. 696166000

2.613396000

1. 621725000

2.448258000

$-0.739766000$

$-1.748832000$

$-0.666812000$

$-1.636887000$

$-0.271187000$

0.321937000

$-0.337782000$

1. 349863000

0.284145000

$-0.342418000$

$-1.458997000$

$-0.699953000$

$-1.941798000$

$-2.227429000$

$-2.135348000$ 


$\begin{array}{ll}\mathrm{H} & 2.261566000 \\ \mathrm{H} & 3.788655000 \\ \mathrm{H} & 3.533879000 \\ \mathrm{C} & 3.465951000 \\ \mathrm{C} & 2.848364000 \\ \mathrm{H} & 2.697466000 \\ \mathrm{H} & 1.875904000 \\ \mathrm{H} & 3.513281000 \\ \mathrm{C} & 4.834291000 \\ \mathrm{H} & 5.524019000 \\ \mathrm{H} & 5.313465000 \\ \mathrm{H} & 4.752284000 \\ \mathrm{C} & 3.652452000 \\ \mathrm{H} & 2.693696000 \\ \mathrm{H} & 4.112713000 \\ \mathrm{H} & 4.313525000\end{array}$
1.051188000
0.152130000
1. 660725000
$-0.365974000$
$-1.267906000$
$-2.300777000$
$-0.881279000$
$-1.318716000$
$-0.933006000$
$-0.967894000$
$-0.313245000$
$-1.960112000$
1.051512000
1. 491315000
1.738727000
1. 023281000

-2.858355000
-2.674992000
-1.771957000
0.765298000
1.851842000
1.497728000
2.196705000
2.733223000
0.337693000
1.200781000
-0.438574000
-0.055152000
1.339254000
1.657427000
0.610712000
2.224549000

$-0.285100000$

$-1.470620000$

1. 153740000

$-0.055920000$

0.098270000

1.148230000

$-1.083430000$

$-1.966630000$

2. 405140000

2. 851960000

3.191860000

2. 161140000

1.144730000

1. 991420000

0.075240000

0.062280000

$-1.055330000$

$-1.918060000$

$-1.775060000$

$-2.729900000$

$-0.999480000$

$-1.153980000$

0.228690000

1. 036740000

1. 133140000

2. 009290000

0.708400000

$-1.093470000$

$-2.117970000$

$-2.769680000$

$-2.243600000$

$-2.454900000$

0.619030000

0.313610000

0.288830000

1. 712830000

0.312540000

$-0.507540000$

$-1.585240000$

$-0.380760000$

$-0.179000000$

0.117710000

0.440370000

0.711290000

$-0.937700000$

1. 802550000 


$\begin{array}{lrrr}\mathrm{H} & -2.625290000 & 1.215870000 & 1.985480000 \\ \mathrm{H} & -3.118110000 & -0.435070000 & 2.441250000 \\ \mathrm{H} & -4.349730000 & 0.811350000 & 2.144620000\end{array}$

TS-1a-exo' : $E=-1200.418138$

$\mathrm{Si}$

0

0

$\mathrm{N}$

N

B

C

$\mathrm{H}$

C

$\mathrm{H}$

$\mathrm{H}$

$\mathrm{H}$

C

$\mathrm{H}$

C

$\mathrm{H}$

C

$\mathrm{H}$

C

$\mathrm{H}$

C

$\mathrm{H}$

C

C

$\mathrm{H}$

$\mathrm{H}$

$\mathrm{H}$

C

C

$\mathrm{H}$

$\mathrm{H}$

$\mathrm{H}$

C

$\mathrm{H}$

$\mathrm{H}$

$\mathrm{H}$

C

C

$\mathrm{H}$

$\mathrm{H}$

$\mathrm{H}$

C

$\mathrm{H}$

$\mathrm{H}$

$\mathrm{H}$

C

$\mathrm{H}$

$\mathrm{H}$

$\mathrm{H}$
$-1.598164000$

1.671847000

3.537965000

$-0.288593000$

2. 490984000

0.356541000

0.054737000

$-0.315892000$

0.226193000

$-0.481412000$

$-0.049852000$

1. 225857000

1.305118000

1.899941000

0.941199000

1. 267371000

0.337718000

0.220579000

2. 210685000

2. 366888000

2. 765098000

3.475203000

3. 009918000

2. 520017000

2. 110804000

3.550755000

1. 918553000

2. 074750000

$-1.676674000$

$-2.056537000$

$-2.359253000$

$-0.683000000$

$-1.250804000$

$-0.174747000$

$-1.752425000$

$-1.620794000$

$-3.239979000$

$-3.477183000$

$-3.551895000$

$-2.669524000$

$-4.423415000$

$-4.392725000$

$-5.355560000$

$-4.261842000$

$-4.488249000$

$-3.193968000$

$-2.398770000$

$-3.028687000$

$-4.152330000$
$-0.777062000$

$-1.853791000$

$-0.055131000$

0.489804000

$-1.237962000$

1.056598000

1.059923000

0.548717000

0.558224000

$-0.255324000$

1. 405244000

0.232810000

2. 242873000

2. 693589000

3. 071849000

4. 114351000

2.450002000

2. 937948000

0.469104000

0.835656000

1.036168000

1. 861168000

$-0.086678000$

$-2.542220000$

$-2.483516000$

$-2.926363000$

$-3.225865000$

$-0.991732000$

$-1.544193000$

$-0.858987000$

$-2.408304000$

$-1.905056000$

$-2.157407000$

$-2.368086000$

$-3.073744000$

$-1.938542000$

0.133935000

1. 243301000

0.843274000

1. 992982000

1.772961000

$-0.889927000$

$-0.396752000$

$-1.701434000$

$-1.350765000$

0.769362000

1. 526772000

0.022383000

1. 273734000
$-0.142184000$

$-1.999571000$

1. 762217000

$-0.132762000$

0.076162000

1.025804000

$-1.364441000$

$-2.253615000$

2. 518567000

2. 710133000

3.170129000

2. 858622000

0.697318000

1. 498559000

$-0.403809000$

$-0.471240000$

$-1.472332000$

$-2.442688000$

$-1.466275000$

$-2.478290000$

$-0.293486000$

$-0.289274000$

0.670109000

0.699410000

1.717909000

0.763604000

0.087064000

$-1.241472000$

$-1.859427000$

$-2.632466000$

$-1.819559000$

$-2.167394000$

1. 090530000

1. 156916000

0.740190000

2.102224000

0.257397000

$-0.786020000$

$-1.810300000$

$-0.774476000$

$-0.573369000$

0.199076000

0.425038000

0.934513000

$-0.797811000$

1. 659633000

1.741381000

2.452606000

1. 879504000

$-0.048263000$

$-1.682236000$

1. 615214000

$-0.133683000$

0.090964000

0.931622000

$-1.466603000$ 


\begin{tabular}{|c|c|c|c|}
\hline $\mathrm{H}$ & -0.222578000 & 0.217870000 & -2.274027000 \\
\hline $\mathrm{C}$ & 0.124992000 & 0.905348000 & 2.483354000 \\
\hline $\mathrm{H}$ & -0.659719000 & 0.213848000 & 2.810541000 \\
\hline $\mathrm{H}$ & -0.071502000 & 1.890710000 & 2.941882000 \\
\hline $\mathrm{H}$ & 1.087574000 & 0.569070000 & 2.911285000 \\
\hline $\mathrm{C}$ & 1.455237000 & 2.103379000 & 0.392426000 \\
\hline $\mathrm{H}$ & 1.956499000 & 2.685626000 & 1.175579000 \\
\hline C & 0.802979000 & 2.953067000 & -0.671891000 \\
\hline $\mathrm{H}$ & 0.828487000 & 4.045230000 & -0.626057000 \\
\hline $\mathrm{C}$ & 0.196115000 & 2.261549000 & -1.647891000 \\
\hline $\mathrm{H}$ & -0.307957000 & 2.703900000 & -2.509417000 \\
\hline C & 1.796274000 & 0.384756000 & -1.454858000 \\
\hline $\mathrm{H}$ & 2.222735000 & 0.564464000 & -2.452156000 \\
\hline $\mathrm{C}$ & 2.499095000 & 1.182644000 & -0.340231000 \\
\hline $\mathrm{H}$ & 3.329354000 & 1.797190000 & -0.720374000 \\
\hline C & 3.078275000 & 0.129010000 & 0.592807000 \\
\hline C & 3.074059000 & -2.366117000 & 0.770759000 \\
\hline $\mathrm{H}$ & 2.301057000 & -2.616276000 & 1.515266000 \\
\hline $\mathrm{H}$ & 4.035101000 & -2.245304000 & 1.287191000 \\
\hline $\mathrm{H}$ & 3.127508000 & -3.172607000 & 0.028474000 \\
\hline $\mathrm{C}$ & 2.009195000 & -1.077499000 & -1.087836000 \\
\hline $\mathrm{C}$ & -1.746101000 & -1.731719000 & -1.694534000 \\
\hline $\mathrm{H}$ & -2.111021000 & -1.097275000 & -2.517339000 \\
\hline $\mathrm{H}$ & -2.452743000 & -2.570081000 & -1.584251000 \\
\hline $\mathrm{H}$ & -0.766436000 & -2.144979000 & -1.978880000 \\
\hline C & -1.257116000 & -2.112514000 & 1.274523000 \\
\hline $\mathrm{H}$ & -0.278453000 & -2.574933000 & 1.065565000 \\
\hline $\mathrm{H}$ & -2.018606000 & -2.908509000 & 1.237047000 \\
\hline $\mathrm{H}$ & -1.236017000 & -1.711836000 & 2.297244000 \\
\hline $\mathrm{C}$ & -3.270153000 & 0.119213000 & 0.276950000 \\
\hline C & -3.460201000 & 1.183662000 & -0.822870000 \\
\hline $\mathrm{H}$ & -3.521464000 & 0.735949000 & -1.828320000 \\
\hline $\mathrm{H}$ & -2.634885000 & 1.914471000 & -0.828364000 \\
\hline $\mathrm{H}$ & -4.399152000 & 1.743103000 & -0.658314000 \\
\hline C & -4.438769000 & -0.886829000 & 0.228730000 \\
\hline $\mathrm{H}$ & -5.399406000 & -0.367511000 & 0.400122000 \\
\hline $\mathrm{H}$ & -4.346195000 & -1.664372000 & 1.005442000 \\
\hline $\mathrm{H}$ & -4.511932000 & -1.393087000 & -0.747392000 \\
\hline $\mathrm{C}$ & -3.267064000 & 0.818316000 & 1.648491000 \\
\hline $\mathrm{H}$ & -2.477221000 & 1.581661000 & 1.718972000 \\
\hline $\mathrm{H}$ & -3.125083000 & 0.106974000 & 2.478487000 \\
\hline $\mathrm{H}$ & -4.232629000 & 1.329698000 & 1.816609000 \\
\hline
\end{tabular}

